Published in final edited form as:

Cochrane Database Syst Rev. ; (2): CD002229. doi:10.1002/14651858.CD002229.pub2.

\title{
Hormone replacement therapy for preventing cardiovascular disease in post-menopausal women
}

\author{
Rafael Gabriel Sanchez ${ }^{1}$, Luis Maria Sanchez Gomez ${ }^{2}$, Loreto Carmona ${ }^{3}$, Marta Roqué i \\ Figuls $^{4}$, and Xavier Bonfill Cosp ${ }^{5}$ \\ 1 Unidad de Investigacion, Hospital Universitario de la Princesa, Madrid, Spain \\ ${ }^{2}$ Agencia Evaluacion Tecnologias Sanitarias de Galicia, Servico Galego de Salude, A Coruna, \\ Spain \\ ${ }^{3}$ Rheumatology Organization, Madrid, Spain \\ ${ }^{4}$ Centro Cochrane Iberoamericano. CIBER Epidemiología y Salud Pública (CIBERESP), Spain., \\ Hospital de la Santa Creu i Sant Pau, Barcelona, Spain \\ ${ }^{5}$ CIBER Epidemiología y Salud Pública (CIBERESP), Spain. Centro Cochrane Iberoamericano, \\ Hospital de la Santa Creu i Sant Pau, Barcelona, Spain
}

\begin{abstract}
Background-There is apparently compelling evidence, from observational studies, that hormone replacement therapy (HRT) may have benefits in reducing cardiovascular events in postmenopausal women. However, these observational data are subject to biases and confounding and require support from formally designed randomised controlled trials of the effects of HRT on cardiovascular disease risk.
\end{abstract}

\footnotetext{
Copyright $\odot 2009$ The Cochrane Collaboration. Published by John Wiley \& Sons, Ltd

Contact address: Rafael Gabriel Sanchez, Unidad de Investigacion, Hospital Universitario de la Princesa, Diego De Leon 62, Planta 9,

Madrid, 28006, Spain.rgabriel.hulp@salud.madrid.org.rgsanchez@hup.es.

Editorial group: Cochrane Heart Group.

Publication status and date: Edited (no change to conclusions), published in Issue 1, 2009.

Review content assessed as up-to-date: 31 January 2005.

CONTRIBUTIONS OF AUTHORS

Rafael Gabriel: participated in developing the protocol; writing the grant application; retrieving papers; data interpretation, and writing the review. Dr Gabriel is the guarantor of this review.

Loreto Carmona: participated in data extraction; appraising the quality of studies; data analysis; data interpretation; writing the review; and entering the review into RevMan.

Marta Roque: participated in developing the protocol; data extraction; appraising the quality of studies; data analysis; data interpretation; writing the review; and entering the review into RevMan.

Luis Maria Sánchez Gómez: participated in developing the protocol; retrieving papers; data extraction; appraising the quality of studies; data management, data interpretation and writing the review.

Margaret Burke: participated in retrieving papers and correcting the search strategy.

Xavier Bonfill: participated in developing the protocol; writing the grant application; data interpretation, and writing the review.

DECLARATIONS OF INTEREST

None known

NOTES

The Peninsula Technology Assessment Group (PenTAG) at Peninsula Medical School, Exeter, UK and the Cochrane Heart Group have been awarded a 3-year grant from the National Institute for Health Research to update existing Cochrane systematic reviews relevant to public health, primary care and rehabilitation.

This review is scheduled to be updated in the first year of the program. Publication of the updated review is anticipated by issue 2, 2009 at the latest.
} 
Objectives-To assess the effects of HRT for the primary and secondary prevention of cardiovascular diseases in post-menopausal women.

Search methods-We searched MEDLINE (1998 to December 2002)), EMBASE (1998 to December 2002), the Cochrane Controlled Trials Register (CCTR) (Issue 4 2002), the National Research Register (1998 to present), ClinicalTrials.gov (1998 to present), and the database of Spanish Clinical Trials (1998 to present) and reference lists of articles.

Selection criteria-Randomised controlled trials comparing HRT with controls (placebo or no treatment) with a minimum follow up of 6 months for treating or preventing cardiovascular disease in postmenopausal women with or without cardiovascular disease.

Data collection and analysis-Three independent reviewers extracted information from the articles, solving discrepancies by consensus. All outcomes studied were dichotomous. Risk ratios and 95\% confidence intervals (CI) were calculated for each study and plotted. Random effects meta-analysis was used in efficacy outcomes (cardiovascular events) and fixed-effects metaanalysis in variables regarding side effects (deep venous thrombosis).

Main results-No protective effect of HRT was seen for any of the cardiovascular outcomes assessed: all cause mortality, cardiovascular death, non-fatal MI, venous thromboemboli or stroke. Higher risks of venous thromboembolic events (Relative risk (RR) 2.15, 95\% CI 1.61 to 2.86), pulmonary embolus (RR 2.15, 95\% CI 1.41 to 3.28), and stroke (RR 1.44, 95\% CI 1.10 to 1.89) was found in those randomised to HRT compared with placebo. No substantial heterogeneity (p $<0.1)$ was detected in any of the outcomes studied.

Authors' conclusions-At present, a recommendation for initiating HRT for the reason of preventing cardiovascular events in post-menopausal women (with or without cardiovascular disease) should not be made. Women with other risk factors for venous thromboembolic events should be discouraged from using HRT if the sole goal is to prevent cardiovascular events.

\section{Medical Subject Headings (MeSH)}

*Estrogen Replacement Therapy; Cardiovascular Diseases [*prevention \& control]; Hormone Replacement Therapy; Postmenopause

\section{MeSH check words}

Female; Humans

\section{BACKGROUND}

Post-menopausal women are at higher risk of cardiovascular disease (CVD) than their younger counterparts. Age at menopause, a measure of the exposure to endogenous oestrogen, is a fair predictor of cardiovascular disease mortality (de Klejin 2002), and it is biologically plausible that exogenous oestrogens might be cardio-protective (Wenger 2000). There have been many observational studies on the use of Hormone Replacement Therapy (HRT) in post-menopausal women in recent years. The potential benefits claimed for HRT include a wide variety of diseases in women, from osteoporosis to dementia, and a special interest exists in the prevention of coronary heart disease. Observational studies have 
consistently found that use of HRT is protective against CHD, with a meta-analysis of observational studies yielding a summary relative risk for ever use of HRT of 0.56 (95\% confidence interval (CI) 0.50 to 0.61 ) (Stampfer 1991). Although the bulk of evidence from observational studies suggests that the use of exogenous oestrogens is cardio-protective for post-menopausal women (Grodstein 1996), the efficacy and effectiveness of HRT in the prevention of cardiovascular diseases in unselected women is not yet clear. Evidence from observational studies is difficult to interpret due to variation in the hormones used, the dose and mode of administration. But above all women willing to take hormones after menopause may differ substantially from those who do not, resulting in seriously confounded estimates of effects of HRT (Lawlor 2004), as well as adding to a general healthy cohort effect (Petitti 1994).

There are some data on intermediate cardiovascular outcomes from clinical trials, the most important of which is the PEPI trial. In this randomised controlled trial 875 healthy postmenopausal women were randomised to receive either placebo or oestrogens, either alone or combined with progestatins. All hormone regimens improved the coronary risk profile at three years, with some adverse effects, mainly endometrial hyperplasia in women with intact uterus. However, an important question remained: was the improvement in cardiovascular risk factors translated into a lower cardiovascular mortality? Although this was widely accepted, it remained to be examined in clinical trials.

In parallel with the PEPI trial (PEPI), the first randomised controlled trial (RCT) to primarily assess the cardio-protective effect of HRT in women with diagnosed cardiovascular disease showed no benefit (HERS). In response to the results from the HERS trial (HERS), many investigators claimed that women included in the trial were not healthy, all had a diagnosis of coronary heart disease (despite this being a design feature of the HERS trial intended to maximise event rates, thereby making detection of any beneficial effect easier) and would not have been put on HRT by any thoughtful clinician as the relevance of using data gathered from observational studies of HRT to predict a reduction in cardiovascular risk is questionable without other supporting evidence. Attention has been drawn to the difficulties women and their doctors face in attempting to deal with contradictory findings (Anon 2004). Although systematic reviews of this topic have been published, they have either pre-dated publication of the large, recent RCTs (Hemminki 1997), or have not been systematic in their conduct (Beral 2002). A systematic review focused on the effects of HRT on cardiovascular outcomes therefore seemed to be a reasonable approach to clarify the growing data on this topic. Material from this systematic review has contributed to an overview of the effects of HRT on a wide range of clinical outcomes (HRT Study Group 2005), which may be of interest to readers of this systematic review.

\section{OBJECTIVES}

To determine the effects of HRT in post-menopausal women with and without pre-existing cardiovascular disease for the prevention of cardiovascular diseases. 


\section{METHODS}

\section{Criteria for considering studies for this review}

Types of studies-Randomised controlled trials comparing HRT with controls (placebo or no treatment) in post-menopausal women with a duration of follow up of 6 months or longer, with primary or secondary outcomes of cardiovascular events.

In the original protocol, prospective longitudinal observational studies (with at least 1000 post-menopausal women at baseline with a minimum of 1 year follow-up), were to be included but with the extent of randomised trial evidence, this aspect of the work was abandoned.

Types of participants-Post-menopausal women (spontaneous or induced cessation of menstrual bleeding for a continuous period of 6 or more months) with or without evidence of cardiovascular disease.

\section{Types of interventions}

- Hormone Replacement Therapy (HRT), either with oestrogens alone or in different combinations with progestogens;

- Placebo (control);

- No treatment (open control.

\section{Types of outcome measures}

\section{Primary outcomes}

- Deaths from any cause;

- Cardiovascular deaths;

- Non-fatal acute myocardial infarction;

- Stroke;

- Combined cardiovascular events outcomes: angina, acute myocardial infarction, venous thromboembolism, stroke and revascularizations (coronary by-pass, angioplasty, coronary stent).

\section{Secondary outcomes}

- Pulmonary emboli;

- Venous thromboemboli (pulmonary emboli plus deep venous thromboses).

In terms of possible harms, data on gynaecological cancers were abstracted from the trials included in addition to the secondary outcomes listed above.

The following outcomes appeared in the protocol but were not considered finally in the analysis:

- $\quad$ side effects and tolerability of treatment; 
- intermediate cardiovascular outcomes (blood pressure, blood cholesterol, and coagulation factors);

- quality of life measures.

\section{Search methods for identification of studies}

The latest issue (Issue 4 2002) of the Cochrane Controlled Trials Register (CCTR) on The Cochrane Library was searched using the strategy outlined below. This was adapted appropriately for searching MEDLINE (1998 to present), EMBASE (1998-present), and the database of Spanish Clinical Trials. In addition, a standard RCT filter was used for MEDLINE (Dickersin 1994) and EMBASE (Lefebvre 1996). The reference lists of relevant papers were checked. The National Research Register and ClinicalTrials.gov were searched for any ongoing trial on $\mathrm{CV}$ diseases.

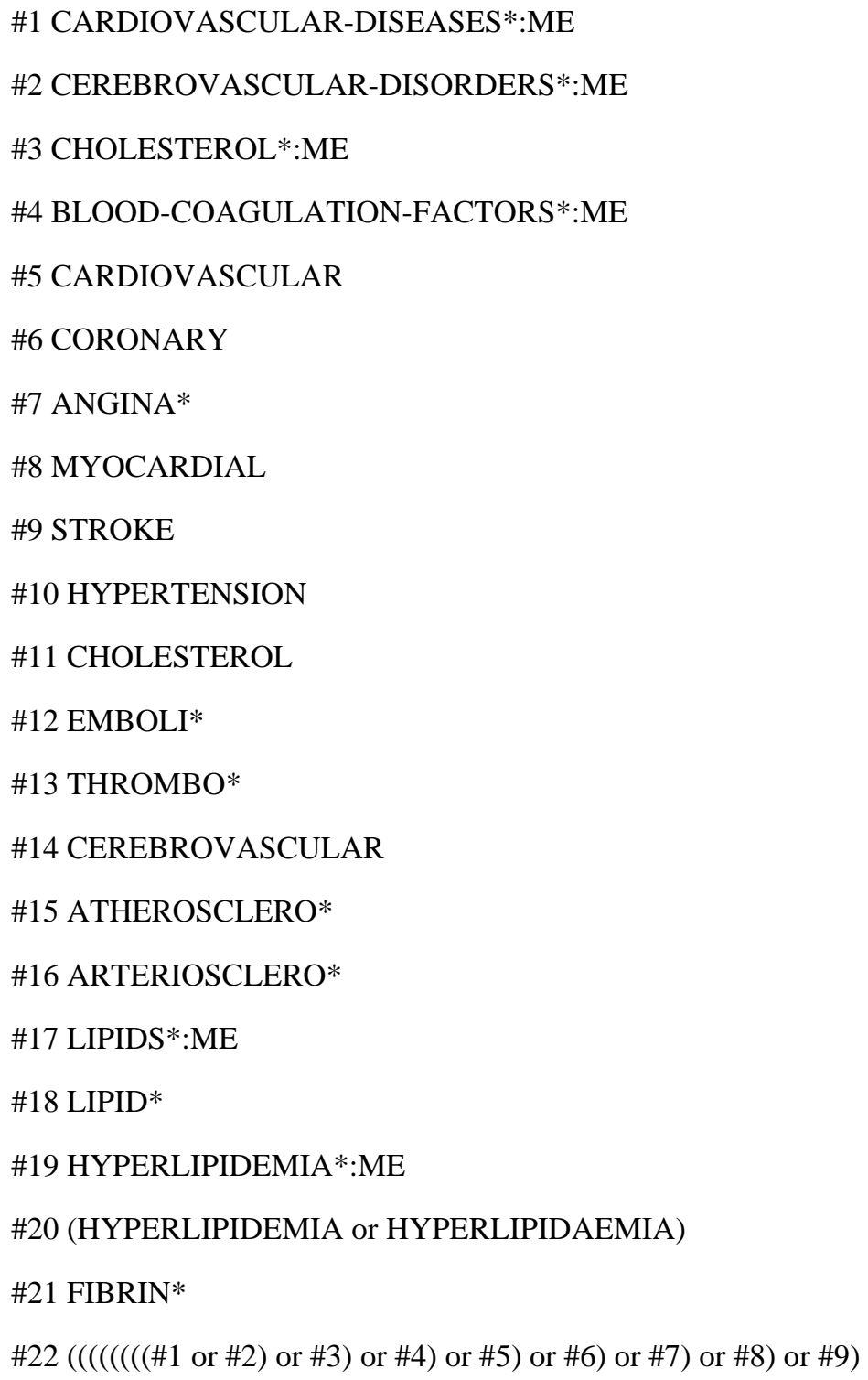




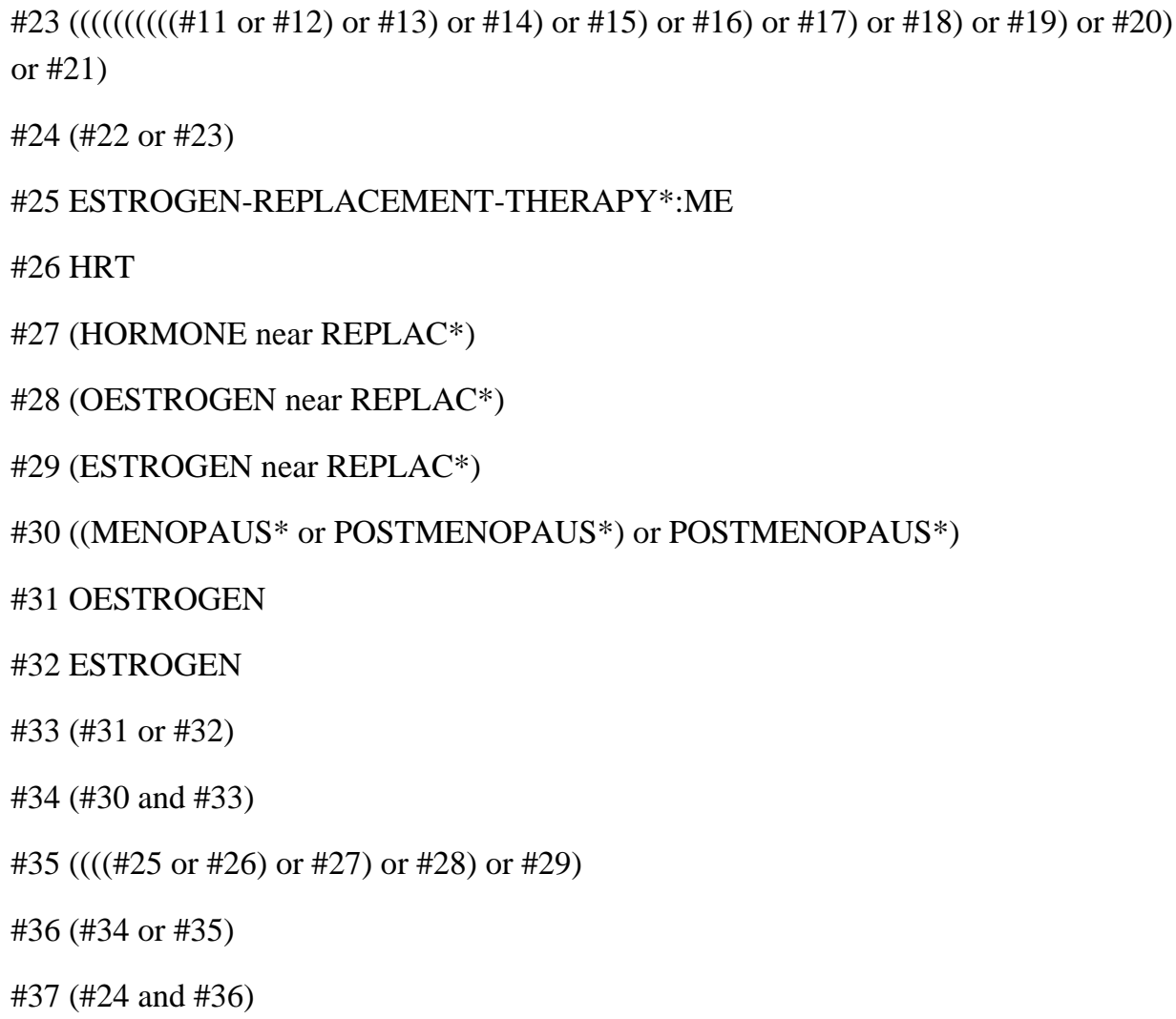

\section{Data collection and analysis}

References for all articles identified from the search strategy were stored and managed within a bibliographic database (Reference Manager 8.5). All titles were screened by two of the reviewers (LCO, LMSG) independently in order to discard those clearly unrelated to the aim of the study.

\section{Inclusion of articles}

Inclusion Criteria: Only those articles meeting the following criteria were considered for closer assessment:

1. Clinical trials according to the Cochrane Collaboration criteria, i.e. studies using random allocation methods (explicit or implicit) of individuals in 2 or more groups of treatment (including the control group or placebo group);

2. Hormonal replacement treatments administered alone or in combination;

3. Inclusion of CV outcomes of relevance for post-menopausal women.

Exclusion Criteria: According to the inclusion criteria mentioned above, trials with only surrogate end-points (i.e. electrocardiographic changes, symptoms, blood pressure, or biochemical changes), reviews, open trials, and animal studies were excluded from further examination. 
Data Extraction: A specific questionnaire for data extraction was designed to record data from the studies selected for inclusion. Three independent reviewers (MRF, LCO and LMSG) extracted the information from the articles. Discrepancies in the results were solved by consensus.

Statistical Analysis: All outcomes studied were dichotomous. Risk ratios and 95\% confidence intervals (CI) were calculated for each study. The results of each RCT were plotted as point estimates with corresponding $95 \%$ confidence intervals. For each outcome, a test of heterogeneity was carried out. Random effects meta-analyses were performed for situations where heterogeneity of effects was apparent. Sensitivity analyses were carried out to explore heterogeneity.

\section{RESULTS}

\section{Description of studies}

See: Characteristics of included studies; Characteristics of excluded studies; Characteristics of ongoing studies.

Of a total of 1497 abstracts of articles and studies retrieved by the different search strategies. 1450 references were discarded. The discarded studies were non-human studies, reviews, editorials, not controlled-trials, laboratory studies, only men included, assessing other drugs different from HRT, language different from English, French, or Spanish. Forty seven (47) articles were assessed in detail. Of these 29 studies (comprising 33 articles) were excluded. Many studies were discarded as the main outcome variables appearing in the abstract were markers, or risk factors, rather than events with no indication that events had been measured. Details for exclusion are given in the excluded studies tables. Ten studies comprising 16 articles were included. Details of included studies are given in the included studies tables.

There was a certain redundancy of publications from the same study, and only the latest versions or that including the newest versions with the outcomes of interest were included.

The ten included studies were randomised placebo controlled trials with a minimum followup of one year (EPAT; ERA; EVTET; HALL; HERS; HERS II; SPRIT 2002; WAWE; WEST;WHI-2002). All studies included postmenopausal women with a mean age between 50 and 79. Eight studies were focused on secondary prevention of cardiovascular events (ERA; EVTET; HALL; HERS; HERS II; SPRIT 2002; WAWE; WEST), and two on primary prevention (EPAT; WHI-2002) in persons with one cardiovascular risk factor (hypercholesterolemia)(EPAT). The EPAT study (EPAT) was focused in an intermediate outcome of CV disease, carotid artery intima-media thickness, but also included CV events as secondary outcomes, and the follow-up period was extensive. The EVTET study (EVTET) was focused on venous thromboembolism for women with previous venous thromboembolism.

We also found four ongoing studies at the time of our search which will be included in an update of this review.(PHASE; NHLBI; WHISP; WISDOM). Of these, WHISP has now been completed and publications are expected. WISDOM has been stopped early due to 
scientific and practical concerns (White 2002). PHASE has now reported, demonstrating an increased event rate in those allocated to transdermal HRT; the relative risk was 1.29 (95\% confidence interval 0.84 to $1.95, \mathrm{P}=0.24$ ) (Clarke 2002). Finally, the NHLBI trial of short term HRT in acute unstable angina has reported 6 month clinical outcomes demonstrating no difference in outcomes between those randomised to either HRT regimen or placebo (Schulman 2002).

\section{Risk of bias in included studies}

All RCTs included yielded a score of five in the Jadad's scale, except for the ERA and Hall's study, with a score of four (Jadad 1996). The extension of follow-up was adequate, and, despite losses of patient, there were efforts to measure the outcomes by secondary approaches other than by visits (clinical records, emergency rooms and intensive care units records, mortality registries).

\section{Effects of interventions}

Overall, information on 12,353 women randomised to HRT were compared with 11930 women randomised to placebo, with an average duration of follow up of 5 years. The plots show the combined effect of HRT on cardiovascular events and side effects. No substantial heterogeneity $(\mathrm{p}<0.1)$ was detected in any of the outcomes studied.

When considering all the trials (primary and secondary prevention) togetherNo protective effect of HRT was seen regarding any of the CV outcomes assessed: death from all causes (Relative risk $(\mathrm{RR})=1.06$; 95\% CI 0.94 to 1.19); death from CV causes only $(\mathrm{RR}=1.04 ; 95 \% \mathrm{CI} 0.85$ to 1.26$)$; non-fatal MI $(\mathrm{RR}=1.11 ; 95 \%$ CI 0.95 to 1.28$)$ or combined $\mathrm{CV}$ events ( $\mathrm{RR}=1.04 ; 95 \% \mathrm{CI} 0.96$ to 1.14$)$. In contrast, a higher risk of venous thromboembolic events (including pulmonary embolus) was observed in the whole population, with a $\mathrm{RR}=2.13$ (95\% CI 1.68 to 2.70$)$ or pulmonary embolus only with a $\mathrm{RR}=2.14$ (95\% CI 1.49 to 3.07) and stroke with a $\mathrm{RR}=1.25$ (95\% CI 1.07 to 1.45 ) related to HRT. Over $60 \%$ of the weight was contributed by only two trials, HERS and HERS II.

When considering only the primary prevention trials-No protective effect of HRT was seen regarding any of the $\mathrm{CV}$ outcomes assessed: death from all causes $(\mathrm{RR}=1.00$; 95\% CI 0.984 to 1.21$)$; death from CV causes only ( $\mathrm{RR}=1.16$; $95 \% \mathrm{CI} 0.70$ to 1.92$)$. In contrast, a higher risk of venous thromboembolic events (including pulmonary embolus) was observed in the whole population, with a $\mathrm{RR}=2.15$ (95\% CI 1.61 to 2.86 ) or pulmonary embolus only with a $\mathrm{RR}=2.15$ (95\% CI 1.41 to 3.28$)$ or combined $\mathrm{CV}$ events with a $\mathrm{RR}=$ 1.20 (95\% CI 1.05 to 1.36$)$ or non-fatal MI with a RR $=1.32$ (95\% CI 1.02 to 1.71$)$ and stroke with a RR $=1.44$ (95\% CI 1.10 to 1.89 ) related to HRT. As only 1 event was observed in EPAT, these findings are essentially those for WHI-2002, and this applies to all subsequent primary prevention findings.

When considering only the secondary prevention trials-No protective effect of HRT was seen regarding any of the CV outcomes assessed: death from all causes ( $\mathrm{RR}=$ 1.08; 95\% CI 0.94 to 1.25$)$; death from CV causes only ( $\mathrm{RR}=1.00 ; 95 \% \mathrm{CI} 0.82$ to 1.23 ); 
non-fatal MI ( $\mathrm{RR}=1.01 ; 95 \% \mathrm{CI} 0.85$ to 1.20$)$; stroke ( $\mathrm{RR}=1.15 ; 95 \% \mathrm{CI} 0.97$ to 1.36 ) or combined $\mathrm{CV}$ events $(\mathrm{RR}=0.96 ; 95 \%$ CI 0.89 to 1.04$)$.

In contrast, a higher risk of venous thromboembolic events (including pulmonary embolus) was observed in the whole population, with a $\mathrm{RR}=2.03$ (95\% CI 1.36 to 3.04) or pulmonary embolus only with a RR=2.07 (95\% CI 1.04 to 4.10) related to HRT.

When considering only the oestrogen alone trials (in primary or secondary prevention)-No protective effect of HRT was seen regarding any of the CV outcomes assessed: death from all causes ( $\mathrm{RR}=0.96$; $95 \%$ CI 0.69 to 1.33); death from CV causes only ( $\mathrm{RR}=0.7095 \%$ CI 0.44 to 1.12$)$; non-fatal $\mathrm{MI}(\mathrm{RR}=1.26$; $95 \%$ CI 0.84 to 1.90$)$; stroke ( $\mathrm{RR}=1.21 ; 95 \% \mathrm{CI} 0.84$ to 1.74$)$; combined $\mathrm{CV}$ events $(\mathrm{RR}=0.74 ; 95 \% \mathrm{CI} 0.53$ to $1.02)$; venous thromboembolic events including pulmonary embolus ( $\mathrm{RR}=0.97 ; 95 \% \mathrm{CI}$ 0.28 to 3.38 ) or pulmonary embolus only ( $\mathrm{RR}=0.98$; $95 \% \mathrm{CI} 0.28$ to 3.39 ).

When considering only the combined HRT (oestrogen + progestogen) trialsNo protective effect of HRT was seen regarding any of the CV outcomes assessed: death from all causes $(\mathrm{RR}=1.07 ; 95 \% \mathrm{CI} 0.94$ to 1.22$)$; death from $\mathrm{CV}$ causes only $(\mathrm{RR}=1.13$; $95 \%$ CI 0.91 to 1.41$)$; non-fatal MI ( $R R=1.08$; $95 \%$ CI 0.92 to 1.27$)$ or combined CV events $(\mathrm{RR}=1.07 ; 95 \%$ CI 0.98 to 1.17$)$. In contrast, a higher risk of venous thromboembolic events (including pulmonary embolus) was observed in the whole population, with a $\mathrm{RR}=2.19$ (95\% CI 1.72 to 2.79 ) or pulmonary embolus only with a $\mathrm{RR}=2.29$ (95\% CI 1.57 to 3.35) and stroke with a $\mathrm{RR}=1.26$ (95\% CI 1.06 to 1.49 ) related to HRT.

A sensitivity analysis was conducted in order to differentiate the effect on primary and secondary prevention of $\mathrm{CV}$ diseases, by taking out of the analysis the two studies aimed to assess primary prevention (EPAT; WHI-2002). No differences with the whole analysis were seen after excluding the EPAT or the WHI studies, although the findings for stroke became less strong ( $\mathrm{RR}=1.16 ; 95 \% \mathrm{CI} 0.94$ to 1.44 ). The risk of pulmonary embolus increased from 2.29 (95\% CI 1.57 to 3.35 ) to 2.88 (95\% CI 1.21 to 6.82 ) when considering only the secondary prevention trials.

\section{DISCUSSION}

Postmenopausal HRT is commonly used to prevent coronary disease in women, based on biological plausibility and observational data (Keating 1999). A meta-analysis in 1992, including data mainly from observational studies, showed an increased life expectancy in women taking HRT, taking adverse events into account (Grady 1992).Hemminki's review of trials published before 1997 found an odds ratio of 1.34 (95\% confidence intervals 0.55 , 3.30) but was too small to be conclusive, and the quality of trials was variable as were the various HRT treatments used (Hemminki 1997). A second report by Hemminki provided an interesting meta-analysis of pooled data from $17 \mathrm{HRT}$ drug applications to the Ministry of Social Affairs and Health of Finland which demonstrated no clear effect of HRT on CV survival (Hemminki 2000). 
This discrepancy between observational and trials data has been the subject of growing debate since the publication of the definitive RCTs (Lawlor 2004). Possible explanations are: different level of risk of the study populations, being higher in general in clinical trials than in observational studies; insufficient adjustment for lifestyle and socio-economic factors in observational studies; insufficient consideration of methodological flaws in experimental studies. In addition, it seems unlikely that the intervention on one single factor, rather than on multiple risk factors, would produce significant changes in the incidence of major CV events.

When considering all the trials, both primary and secondary prevention, the results of the current systematic review do not show protection from subsequent $\mathrm{CV}$ events in postmenopausal women taking HRT. Only two studies included in our analysis were aimed to assess whether postmenopausal women without previous CV disease would benefit from HRT (EPAT, WHI-2002). Nevertheless, the primary outcome of the EPAT study (EPAT) was not CV events, but an intermediate outcome: the intima-media thickness of the carotid artery. The results regarding this outcome favoured HRT, but when CV events were taken into account, no benefits were seen. Another additional study (Angerer 2002) after one year of HRT, failed to demonstrate any slowing of progression of subclinical atherosclerosis (measured as intima-media thickness in carotid arteries) in postmenopausal women at increased risk. It had been hoped that long term outcomes would be available but WISDOM, intended to study women till 2016 has been stopped early owing to scientific and practical reasons (White, 2002).

After the publication of the WHI study (WHI-2002), the question of whether healthy women should take HRT to prevent CV disease seems to be easier to answer. The WHI study is the first randomised primary prevention trial of postmenopausal hormone replacement. The data and safety monitoring board recommended stopping the trial because women receiving HRT had an increased risk of invasive breast cancer. Overall, the results of the WHI study are consistent with the body of evidence on the effects of HRT. Risk of stroke and venous thromboembolism and myocardial infarction were also increased in women assigned to the HRT in the WHI.

\section{AUTHORS' CONCLUSIONS}

\section{Implications for practice}

Treatment with HRT for the sole purpose of preventing CV events in post-menopausal women (with or without $\mathrm{CV}$ disease) is not effective.

Among women taking HRT for other reasons (e.g. menopausal symptoms) but with other risk factors for venous thromboembolic events, HRT should be discouraged as there is evidence that HRT causes an increased risk of these events.

\section{Implications for research}

The need to develop new treatments to control perimenopausal symptoms is essential given these findings from the RCTs. Future observational studies examining effects of treatments should be interpreted cautiously given these findings. 


\section{Acknowledgments}

All authors are members of the Red Temática de Investigación en Medicina Basada en la Evidencia FIS G03/090 and Dr. Rafael Gabriel and Dr. Luis M. Sánchez are members of the Red Temática de Investigación en Epidemiología Cardiovascular:Proyecto ERICE. FIS G03/065. The authors want to thank the personnel at the Iberoamerican Cochrane Centre and the Cochrane Heart Group for their assistance with the preparation of this systematic review.

\section{SOURCES OF SUPPORT}

Internal sources

- Instituto de Salud Carlos III (Contrato Investigadores del SNS), Spain.

- Hospital Universitario de la Princesa (Madrid), Spain.

- Centro Cochrane Iberoamericano (Barcelona), Spain.

- Hospital Universitario la Paz (Madrid), Spain.

- Spanish Foundation of Rheumatology, Spain.

External sources

- Agencia de Evaluació de Tecnologies Mèdiques de Catalunya, Spain.

- $\quad$ Red Tematica de Investigacion en Medicina Basada en la Evidencia FIS G03/090, Spain.

- $\quad$ Red Tematica de Investigacion en Medicina Basada en Epidemiologia Cardiovascular: Proyecto ERICE FIS G03/065, Spain.

\section{CHARACTERISTICS OF STUDIES}

\section{Characteristics of included studies [ordered by study ID]}

EPAT

\begin{tabular}{|c|c|}
\hline Methods & Randomised controlled trial of 2 years of follow-up \\
\hline Participants & 222 post-menopausal women (mean age 62.2 ) without previous coronary artery disease \\
\hline Interventions & $\begin{array}{l}\text { A. 17-beta-estradiol } \\
\text { B. placebo }\end{array}$ \\
\hline Outcomes & $\begin{array}{l}\text { Primary: intima thickness } \\
\text { Secondary: } \\
\text { Major CV events } \\
\text { All causes of death } \\
\text { Side effects }\end{array}$ \\
\hline Notes & $25 \%$ Losses of follow-up. \\
\hline \multicolumn{2}{|l|}{ Risk of bias } \\
\hline Item & Authors judgement \\
\hline Allocation concealment? & A - Adequate \\
\hline
\end{tabular}

ERA

Methods $\quad$ Randomised controlled trial of 3.2 years of follow-up


Participants

Interventions

309 post-menopausal women (mean age 65.8 ) with previous coronary artery disease
A. Estrogen + placebo
B. Estrogen + progestin
C. placebo + placebo

\begin{tabular}{lll}
\hline Outcomes & $\begin{array}{l}\text { All causes of death } \\
\text { Major CV events } \\
\text { CV deaths } \\
\text { Revascularization procedures other CV outcomes } \\
\text { Side effects }\end{array}$ \\
\hline Notes & Losses of follow-up evenly distributed. & \\
\hline Risk of bias & & \\
\hline Item & Authors' judgement & Description \\
\hline Allocation concealment? & Yes & A - Adequate \\
\hline
\end{tabular}

EVTET

\begin{tabular}{lll}
\hline Methods & Randomised controlled trial of 1.3 years of follow-up \\
\hline Participants & 140 women with previous venous thromboembolism \\
\hline Interventions & A) $2 \mathrm{mg}$ stradiol ans 1 mg of norethisterone acetate \\
\hline Outcomes & venous thromboembolism \\
\hline Notes & terminated after report of increased risk of venous thromboembolism \\
\hline Risk of bias & & \\
\hline Item & Authors' judgement & Description \\
\hline Allocation concealment? & Yes & A - Adequate \\
\hline
\end{tabular}

HALL

\begin{tabular}{|c|c|}
\hline Methods & Randomised controlled trial of 1 year of follow-up \\
\hline Participants & 60 post-menopausal women (mean age 59.4) with previous coronary artery disease \\
\hline Interventions & $\begin{array}{l}\text { A. 17-beta-estradiol + Medroxyprogesterone acetate } \\
\text { B. placebo } \\
\text { C. Estrogen + Medroxyprogesterone acetate }\end{array}$ \\
\hline Outcomes & $\begin{array}{l}\text { All causes of death } \\
\text { Major CV events } \\
\text { CV deaths } \\
\text { Revascularization procedures }\end{array}$ \\
\hline Notes & $23 \%$ Losses of follow-up \\
\hline \multicolumn{2}{|l|}{ Risk of bias } \\
\hline Item & Authors' judgement \\
\hline Allocation concealment? & A - Adequate \\
\hline
\end{tabular}

Cochrane Database Syst Rev. Author manuscript; available in PMC 2014 September 15. 
HERS

\begin{tabular}{|c|c|}
\hline Methods & Randomised controlled trial of 3.1 years of follow-up \\
\hline Participants & 2763 post- menopausal women (mean age 66.7 ) with previous coronary artery disease \\
\hline Interventions & $\begin{array}{l}\text { A. Estrogen }+ \text { progestinf } \\
\text { B. placebo + placebo }\end{array}$ \\
\hline Outcomes & $\begin{array}{l}\text { All causes of death } \\
\text { Major CV events } \\
\text { CV deaths } \\
\text { Revascularization procedures other CV outcomes } \\
\text { Side effects }\end{array}$ \\
\hline Notes & Vital status known to all. No losses to follow-up. \\
\hline \multicolumn{2}{|l|}{ Risk of bias } \\
\hline Item & Authors' judgement \\
\hline Allocation concealment? & A - Adequate \\
\hline
\end{tabular}

HERS II

\begin{tabular}{ll}
\hline Methods & $\begin{array}{l}\text { Randomised, blinded, placebo controlled trial of 4.1 years duration (HERS) and follow-up } \\
\text { for } 2.7 \text { years }\end{array}$ \\
\hline Participants & 2321 women consented for follow-up in HERS II \\
\hline Interventions & Open label hormone therapy in HERS II \\
\hline Outcomes & $\begin{array}{l}\text { Primary outcome: Non fatal myocardial infarction and CHD death } \\
\text { Secondary cardiovascular events: coronary revascularization, hospitalisation for unstable } \\
\text { angina or congestive heart failure, ventricular arrhythmia, sudden death, stroke, ischemic } \\
\text { attack, peripheral arterial diseases } \\
\text { Non cardiovascular outcomes: thromboembolics events, cancer and al causes of death }\end{array}$ \\
\hline Notes & Cardiovascular and Non cardiovascular Disease Outcomes from the HERS II \\
\hline Risk of bias & Authors' judgement \\
\hline Item & Yes \\
\hline Allocation concealment? &
\end{tabular}

SPRIT 2002

\begin{tabular}{lc}
\hline Methods & Randomised controlled trial of 2 years of follow-up \\
\hline Participants & 1017 postmenopausal women with first myocardial infarction \\
\hline Interventions & A. oestrogen (2 mg oestradiol) \\
& B. Placebo \\
\hline Outcomes & Major CV events \\
\hline CV deaths \\
\hline Rotes
\end{tabular}

Cochrane Database Syst Rev. Author manuscript; available in PMC 2014 September 15. 


\begin{tabular}{lll} 
Item & Authors' judgement & Description \\
\hline Allocation concealment? & Yes & A - Adequate \\
\hline
\end{tabular}

\section{WAWE}

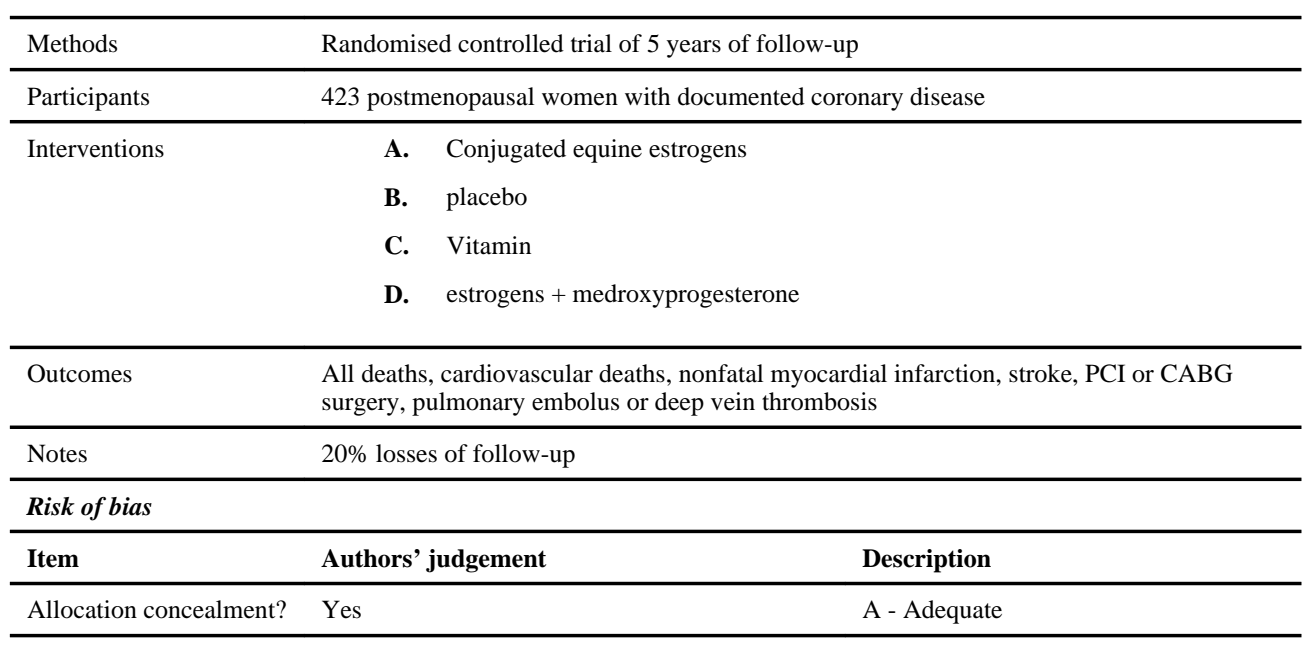

\section{WEST}

\begin{tabular}{|c|c|}
\hline Methods & Randomised controlled trial of 3.1 years of follow-up \\
\hline Participants & 664 post-menopausal women (mean age 71 ) with previous ischemic stroke \\
\hline Interventions & $\begin{array}{l}\text { A. 17-beta-estradiol } \\
\text { B. placebo }\end{array}$ \\
\hline Outcomes & $\begin{array}{l}\text { All causes of death } \\
\text { Major CV events } \\
\text { CV deaths } \\
\text { Side effects }\end{array}$ \\
\hline Notes & No losses to follow-up \\
\hline \multicolumn{2}{|l|}{ Risk of bias } \\
\hline Item & Authors' judgement \\
\hline Allocation concealment? & A - Adequate \\
\hline
\end{tabular}

WHI-2002

\begin{tabular}{lc}
\hline Methods & Randomised controlled trial of 5.2 years of follow-up \\
\hline Participants & 16608 postmenopausal women aged 50-79 years with an intact uterus at baseline \\
\hline Interventions & A. $\quad$ equine estrogens + Medroxyprogesterone acetate
\end{tabular}

Cochrane Database Syst Rev. Author manuscript; available in PMC 2014 September 15. 
B. placebo

\begin{tabular}{lll}
\hline Outcomes & $\begin{array}{l}\text { Primary outcomes: non fatal myocardial infarction and CHD death } \\
\text { Secondary outcomes: stroke, pulmonary embolism, cancer and all causes of death }\end{array}$ \\
\hline Notes & & \\
\hline Risk of bias & Authors' judgement & Description \\
\hline Item & B - Unclear \\
\hline Allocation concealment? & Unclear & \\
\hline
\end{tabular}

\section{Characteristics of excluded studies [ordered by study ID]}

\begin{tabular}{|c|c|}
\hline Study & Reason for exclusion \\
\hline AITKEN & Did not record clinical outcomes. \\
\hline ALOIA & Did not record clinical outcomes. \\
\hline ANGERER & Did not record clinical outcomes. \\
\hline Barret Connor & $\begin{array}{l}\text { The intervention can not be considered "sensu stricto" HRT (women are treated with } \\
\text { Raloxifene, a new oestrogen receptor modulator) }\end{array}$ \\
\hline CHART & Did not record clinical outcomes. \\
\hline CHRISTENSEN & Did not record clinical outcomes. \\
\hline CHRISTIANSEN (a) & Did not record clinical outcomes. \\
\hline CHRISTIANSEN (b) & Did not record clinical outcomes. \\
\hline CHRISTIANSEN (c) & Did not record clinical outcomes. \\
\hline COOPE & Did not record clinical outcomes. \\
\hline DERMAN & Did not record clinical outcomes. \\
\hline GALLAGHER & Did not record clinical outcomes. \\
\hline GENANT & Did not record clinical outcomes. \\
\hline HASSAGER & Did not record clinical outcomes. \\
\hline Heckbert 1997 & Case control study \\
\hline JENSEN & Did not record clinical outcomes. \\
\hline LINDSAY & Did not record clinical outcomes. \\
\hline LUFKIN & Did not record clinical outcomes. \\
\hline MARSLEW & Did not record clinical outcomes. \\
\hline MOLANDERM & Did not record clinical outcomes. \\
\hline MUNK-JENSEN & Did not record clinical outcomes. \\
\hline NACHTIGALL & Did not record clinical outcomes. \\
\hline PEPI & Did not record clinical outcomes. \\
\hline RESCH & Did not record clinical outcomes. \\
\hline RIGGS & Did not record clinical outcomes. \\
\hline RIIS & Did not record clinical outcomes. \\
\hline SVENDSEN & Did not record clinical outcomes. \\
\hline TONSTAD & Did not record clinical outcomes. \\
\hline
\end{tabular}




\begin{tabular}{ll}
\hline Study & Reason for exclusion \\
\hline WIMALAWANSA & Did not record clinical outcomes. \\
\hline
\end{tabular}

\section{Characteristics of ongoing studies [ordered by study ID]}

\section{ESPRIT-UK}

\begin{tabular}{lr}
\hline Trial name or title & Oestrogen in the prevention of the Terinfartion Trial \\
\hline Methods & \\
\hline Participants & 1017 postmenopausal women with bfirs Myocardial Infarction \\
\hline Interventions & A. $\quad$ Estrogens \\
& B. Placebo \\
\hline Outcomes & \\
\hline Starting date & 2000 \\
\hline Contact information & Kahn MA \\
\hline Notes & Secondary prevention \\
\hline
\end{tabular}

NHLBI

\begin{tabular}{ll}
\hline Trial name or title & Postmenopausal Hormone Therapy in Unstable Angina \\
\hline Methods & \\
\hline Participants & Postmenopausal women with unstable angina \\
\hline Interventions & A. $\quad$ Estrogen + progestin \\
& B. Estrogen \\
& C. Placebo \\
& Ischemic episodes \\
\hline Outcomes & 1999 \\
\hline Starting date & Steven P Schulman \\
\hline Contact information & John Hopkins Hospital \\
& Baltimore, Maryland USA Tel: +014109557378 \\
\hline Notes & \\
\hline
\end{tabular}

PHASE

\begin{tabular}{lc}
\hline Trial name or title & The Papworth HRT Atherosclerosis Survival Enquiry \\
\hline Methods & \\
\hline Participants & 400 women with ischemic heart disease \\
\hline Interventions & A. $\quad$ Estrogens $+/$ - progestin
\end{tabular}


B. Placebo $+/-$ placebo

\begin{tabular}{ll}
\hline Outcomes & $\begin{array}{l}\text { Death } \\
\text { MI } \\
\text { Hospitalisations for unstable angina in 2 years }\end{array}$ \\
\hline Starting date & \\
\hline Contact information & $\begin{array}{l}\text { Dr. Sarah C Clarke } \\
\text { Box No 76 } \\
\\
\text { Papworth Hospital } \\
\text { CB3 8RE } \\
\text { UK }\end{array}$ \\
\hline Notes & $\begin{array}{l}\text { Secondary prevention } \\
\text { (It should be completed) }\end{array}$ \\
\hline
\end{tabular}

WHISP

\begin{tabular}{ll}
\hline Trial name or title & Women's hormone intervention secondary prevention study \\
\hline Methods & \\
\hline Participants & $4,000-4,800$ postmenopausal women who have recently had myocardial infarction \\
\hline Interventions & HRT \\
\hline Outcomes & All-cause mortality over a period of 4 years \\
\hline Starting date & \\
\hline Contact information & Malcom.Whitehead@ kingshc.nhs.uk \\
\hline Notes & Secondary prevention \\
\hline
\end{tabular}

\section{WISDOM}

\begin{tabular}{ll}
\hline Trial name or title & Women's International Study of Long Duration Oestrogen After Menopause \\
\hline Methods & \\
\hline Participants & 34,000 post-menopausal healthy women \\
\hline Interventions & Estrogen + Progestin \\
\hline Outcomes & $\begin{array}{l}\text { CV events } \\
\text { Side effects } \\
\text { Quality of life }\end{array}$ \\
\hline Starting date & $\begin{array}{l}\text { 2001. } \\
10 \text { years of follow up }\end{array}$ \\
\hline Contact information & M.R.VICKERS@ mds.qmw.ac.uk \\
\hline Notes & Primary and secondary prevention \\
\hline
\end{tabular}




\section{DATA AND ANALYSES}

\section{Comparison 1 \\ HRT vs placebo (in primary prevention)}

\begin{tabular}{|c|c|c|c|c|}
\hline Outcome or subgroup title & No. of studies & No. of participants & Statistical method & Effect size \\
\hline 1 Death (all causes) & 2 & 16830 & $\begin{array}{l}\text { Risk Ratio (M-H, Fixed, } \\
95 \% \text { CI) }\end{array}$ & $1.00[0.84,1.21]$ \\
\hline 2 Death (CV cause) & 2 & 16830 & $\begin{array}{l}\text { Risk Ratio (M-H, Fixed, } \\
95 \% \text { CI) }\end{array}$ & $1.16[0.70,1.92]$ \\
\hline 3 Non-fatal MI & 2 & 16830 & $\begin{array}{l}\text { Risk Ratio (M-H, Fixed, } \\
95 \% \text { CI) }\end{array}$ & $1.32[1.02,1.71]$ \\
\hline 4 Stroke & 2 & 16830 & $\begin{array}{l}\text { Risk Ratio (M-H, Fixed, } \\
95 \% \text { CI) }\end{array}$ & $1.44[1.10,1.89]$ \\
\hline $\begin{array}{l}5 \text { Combined CV events and } \\
\text { outcomes }\end{array}$ & 2 & 16830 & $\begin{array}{l}\text { Risk Ratio (M-H, Fixed, } \\
95 \% \text { CI) }\end{array}$ & $1.20[1.05,1.36]$ \\
\hline 6 Venous thromboembolism & 1 & 16608 & $\begin{array}{l}\text { Risk Ratio (M-H, Fixed, } \\
95 \% \text { CI) }\end{array}$ & $2.15[1.61,2.86]$ \\
\hline 7 Pulmonary embolus & 1 & 16608 & $\begin{array}{l}\text { Risk Ratio (M-H, Fixed, } \\
95 \% \text { CI) }\end{array}$ & $2.15[1.41,3.28]$ \\
\hline
\end{tabular}

Comparison 2

HRT vs placebo (secondary prevention only)

\begin{tabular}{|c|c|c|c|c|}
\hline Outcome or subgroup title & No. of studies & No. of participants & Statistical method & Effect size \\
\hline 1 Death (all causes) & 7 & 7453 & $\begin{array}{l}\text { Risk Ratio (M-H, Fixed, } \\
95 \% \text { CI) }\end{array}$ & $1.08[0.94,1.25]$ \\
\hline 2 Death (CV cause) & 7 & 7453 & $\begin{array}{l}\text { Risk Ratio (M-H, Fixed, } \\
95 \% \mathrm{CI})\end{array}$ & $1.00[0.82,1.23]$ \\
\hline 3 Non-fatal MI & 6 & 7393 & $\begin{array}{l}\text { Risk Ratio (M-H, Fixed, } \\
95 \% \mathrm{CI})\end{array}$ & $1.01[0.85,1.20]$ \\
\hline 4 Stroke & 7 & 7453 & $\begin{array}{l}\text { Risk Ratio (M-H, Fixed, } \\
95 \% \text { CI) }\end{array}$ & $1.15[0.97,1.36]$ \\
\hline $\begin{array}{l}5 \text { Combined CV events and } \\
\text { outcomes }\end{array}$ & 6 & 6433 & $\begin{array}{l}\text { Risk Ratio (M-H, Fixed, } \\
95 \% \mathrm{CI})\end{array}$ & $0.96[0.89,1.04]$ \\
\hline 6 Venous thromboembolism & 7 & 7533 & $\begin{array}{l}\text { Risk Ratio (M-H, Fixed, } \\
95 \% \text { CI) }\end{array}$ & $2.03[1.36,3.04]$ \\
\hline 7 Pulmonary embolus & 5 & 6905 & $\begin{array}{l}\text { Risk Ratio (M-H, Fixed, } \\
95 \% \text { CI) }\end{array}$ & $2.07[1.04,4.10]$ \\
\hline
\end{tabular}

Comparison 3

HRT vs placebo (in primary or secondary prevention)

\begin{tabular}{lcclc}
\hline Outcome or subgroup title & No. of studies & No. of participants & \multicolumn{1}{c}{ Statistical method } & Effect size \\
\hline 1 Death (all causes) & 9 & 24283 & $\begin{array}{l}\text { Odds Ratio (M-H, Fixed, } \\
95 \% \text { CI) }\end{array}$ & 1.06 [0.94, 1.19] \\
2 Death (CV cause) & 9 & 24283 & $\begin{array}{l}\text { Odds Ratio (M-H, Fixed, } \\
95 \% \text { CI) }\end{array}$ & 1.04 [0.85, 1.26] \\
3 Non-fatal MI & 8 & 24223 & $\begin{array}{l}\text { Odds Ratio (M-H, Fixed, } \\
95 \% \text { CI) }\end{array}$ & $1.11[0.95,1.28]$ \\
4 Stroke & 9 & 24283 & $\begin{array}{l}\text { Odds Ratio (M-H, Fixed, } \\
95 \% \text { CI) }\end{array}$ & $1.25[1.07,1.45]$
\end{tabular}




\begin{tabular}{lcccc}
\hline Outcome or subgroup title & No. of studies & No. of participants & \multicolumn{1}{c}{ Statistical method } & Effect size \\
\hline $\begin{array}{l}\text { 5 Combined CV events and } \\
\text { outcomes }\end{array}$ & 8 & 23266 & $\begin{array}{l}\text { Odds Ratio (M-H, Fixed, } \\
95 \% \text { CI) }\end{array}$ & 1.04 [0.96, 1.14] \\
$\begin{array}{l}\text { 6 Venous thromboembolism } \\
7 \text { Pulmonary embolus }\end{array}$ & 8 & 24141 & $\begin{array}{l}\text { Odds Ratio (M-H, Fixed, } \\
95 \% \text { CI) }\end{array}$ & 2.13 [1.68, 2.70] \\
& 6 & 23513 & $\begin{array}{l}\text { Odds Ratio (M-H, Fixed, } \\
95 \% \text { CI) }\end{array}$ & 2.14 [1.49, 3.07] \\
\hline
\end{tabular}

\section{Comparison 4}

Oestrogen vs placebo (in primary and secondary prevention)

\begin{tabular}{|c|c|c|c|c|}
\hline Outcome or subgroup title & No. of studies & No. of participants & Statistical method & Effect size \\
\hline 1 Death (all causes) & 3 & 1903 & $\begin{array}{l}\text { Odds Ratio (M-H, Fixed, } \\
95 \% \text { CI) }\end{array}$ & $0.96[0.69,1.33]$ \\
\hline 2 Death (CV causes) & 3 & 1903 & $\begin{array}{l}\text { Odds Ratio (M-H, Fixed, } \\
95 \% \text { CI) }\end{array}$ & $0.70[0.44,1.12]$ \\
\hline 3 Non-fatal MI & 3 & 1903 & $\begin{array}{l}\text { Odds Ratio (M-H, Fixed, } \\
95 \% \text { CI) }\end{array}$ & $1.26[0.84,1.90]$ \\
\hline 4 Stroke & 3 & 1903 & $\begin{array}{l}\text { Odds Ratio (M-H, Fixed, } \\
95 \% \text { CI) }\end{array}$ & $1.21[0.84,1.74]$ \\
\hline $\begin{array}{l}5 \text { Combined CV events and } \\
\text { outcomes }\end{array}$ & 3 & 888 & $\begin{array}{l}\text { Odds Ratio (M-H, Fixed, } \\
95 \% \text { CI) }\end{array}$ & $0.74[0.53,1.02]$ \\
\hline 6 Venous thromboembolism & 2 & 1681 & $\begin{array}{l}\text { Odds Ratio (M-H, Fixed, } \\
95 \% \text { CI) }\end{array}$ & $0.97[0.28,3.38]$ \\
\hline 7 Pulmonary embolus & 2 & 1681 & $\begin{array}{l}\text { Odds Ratio (M-H, Fixed, } \\
95 \% \text { CI) }\end{array}$ & $0.98[0.28,3.39]$ \\
\hline
\end{tabular}

Comparison 5

Oestrogen vs placebo (in primary prevention)

\begin{tabular}{|c|c|c|c|c|}
\hline $\begin{array}{l}\text { Outcome or } \\
\text { subgroup title }\end{array}$ & No. of studies & No. of participants & Statistical method & Effect size \\
\hline 1 Death (all causes) & 1 & 222 & $\begin{array}{l}\text { Odds Ratio (M-H, Fixed, 95\% } \\
\text { CI) }\end{array}$ & $0.33[0.01,8.20]$ \\
\hline 2 Death (CV cause) & 1 & 222 & $\begin{array}{l}\text { Odds Ratio (M-H, Fixed, } 95 \% \\
\text { CI) }\end{array}$ & $0.33[0.01,8.20]$ \\
\hline 3 Non-fatal MI & 1 & 222 & $\begin{array}{l}\text { Odds Ratio (M-H, Fixed, } 95 \% \\
\text { CI) }\end{array}$ & $1.0[0.06,16.19]$ \\
\hline 4 Stroke & 1 & 222 & $\begin{array}{l}\text { Odds Ratio (M-H, Fixed, } 95 \% \\
\text { CI) }\end{array}$ & $5.09[0.24,107.27]$ \\
\hline $\begin{array}{l}5 \text { Combined CV } \\
\text { events }\end{array}$ & 1 & 222 & $\begin{array}{l}\text { Odds Ratio (M-H, Fixed, } 95 \% \\
\text { CI) }\end{array}$ & $1.51[0.25,9.24]$ \\
\hline
\end{tabular}


Comparison 6

Oestrogen vs placebo (in secondary prevention)

\begin{tabular}{|c|c|c|c|c|}
\hline Outcome or subgroup title & No. of studies & No. of participants & Statistical method & Effect size \\
\hline 1 Death (all causes) & 2 & 1681 & $\begin{array}{l}\text { Odds Ratio (M-H, Fixed, } \\
95 \% \text { CI) }\end{array}$ & $0.97[0.70,1.35]$ \\
\hline 2 Death (CV causes) & 2 & 1681 & $\begin{array}{l}\text { Odds Ratio (M-H, Fixed, } \\
95 \% \text { CI) }\end{array}$ & $0.72[0.45,1.15]$ \\
\hline 3 Non-fatal MI & 2 & 1681 & $\begin{array}{l}\text { Odds Ratio (M-H, Fixed, } \\
95 \% \text { CI) }\end{array}$ & $1.27[0.84,1.92]$ \\
\hline 4 Stroke & 2 & 1681 & $\begin{array}{l}\text { Odds Ratio (M-H, Fixed, } \\
95 \% \text { CI) }\end{array}$ & $1.17[0.81,1.70]$ \\
\hline $\begin{array}{l}5 \text { Combined } \mathrm{CV} \text { events and } \\
\text { outcomes }\end{array}$ & 2 & 666 & $\begin{array}{l}\text { Odds Ratio (M-H, Fixed, } \\
95 \% \text { CI) }\end{array}$ & $0.72[0.51,1.00]$ \\
\hline 6 Venous thromboembolism & 2 & 1681 & $\begin{array}{l}\text { Odds Ratio (M-H, Fixed, } \\
95 \% \text { CI) }\end{array}$ & $0.97[0.28,3.38]$ \\
\hline 7 Pulmonary embolism & 2 & 1681 & $\begin{array}{l}\text { Odds Ratio (M-H, Fixed, } \\
95 \% \text { CI) }\end{array}$ & $0.98[0.28,3.39]$ \\
\hline
\end{tabular}

Comparison 7

Combined therapy vs Placebo (in primary or secondary prevention)

\begin{tabular}{|c|c|c|c|c|}
\hline Outcome or subgroup title & No. of studies & No. of participants & Statistical method & Effect size \\
\hline 1 Death (all causes) & 7 & 22382 & $\begin{array}{l}\text { Odds Ratio (M-H, Fixed, } \\
\text { 95\% CI) }\end{array}$ & $1.07[0.94,1.22]$ \\
\hline 2 Death (CV cause) & 6 & 22380 & $\begin{array}{l}\text { Odds Ratio (M-H, Fixed, } \\
95 \% \text { CI) }\end{array}$ & $1.13[0.91,1.41]$ \\
\hline 3 Non-fatal MI & 5 & 22320 & $\begin{array}{l}\text { Odds Ratio (M-H, Fixed, } \\
95 \% \text { CI) }\end{array}$ & $1.08[0.92,1.27]$ \\
\hline 4 Stroke & 6 & 22380 & $\begin{array}{l}\text { Odds Ratio (M-H, Fixed, } \\
95 \% \text { CI) }\end{array}$ & $1.26[1.06,1.49]$ \\
\hline $\begin{array}{l}5 \text { Combined CV events and } \\
\text { outcomes }\end{array}$ & 6 & 22377 & $\begin{array}{l}\text { Odds Ratio (M-H, Fixed, } \\
95 \% \text { CI) }\end{array}$ & $1.07[0.98,1.17]$ \\
\hline 6 Venous thromboembolism & 6 & 22460 & $\begin{array}{l}\text { Odds Ratio (M-H, Fixed, } \\
95 \% \text { CI) }\end{array}$ & $2.19[1.72,2.79]$ \\
\hline 7 Pulmonary embolus & 4 & 21832 & $\begin{array}{l}\text { Odds Ratio (M-H, Fixed, } \\
95 \% \text { CI) }\end{array}$ & $2.29[1.37,3.33]$ \\
\hline
\end{tabular}

Comparison 8

Combined therapy vs Placebo (in primary prevention)

\begin{tabular}{lcllc}
\hline Outcome or subgroup title & No. of studies & No. of participants & Statistical method & Effect size \\
\hline 1 Death (all causes) & 1 & $\begin{array}{l}\text { Odds Ratio (M-H, } \\
\text { Fixed, 95\% CI) }\end{array}$ & Totals not selected \\
2 Death (CV cause) & 1 & $\begin{array}{l}\text { Odds Ratio (M-H, } \\
\text { Fixed, 95\% CI) }\end{array}$ & Totals not selected \\
$\begin{array}{l}\text { 3 Non-fatal myocardial } \\
\text { infarction }\end{array}$ & 1 & $\begin{array}{l}\text { Odds Ratio (M-H, } \\
\text { Fixed, 95\% CI) }\end{array}$ & Totals not selected
\end{tabular}




\begin{tabular}{|c|c|c|c|c|}
\hline Outcome or subgroup title & No. of studies & No. of participants & Statistical method & Effect size \\
\hline 4 Stroke & 1 & & $\begin{array}{l}\text { Odds Ratio (M-H, } \\
\text { Fixed, } 95 \% \mathrm{CI})\end{array}$ & Totals not selected \\
\hline $\begin{array}{l}5 \text { Combined CV events and } \\
\text { outcomes }\end{array}$ & 1 & & $\begin{array}{l}\text { Odds Ratio (M-H, } \\
\text { Fixed, } 95 \% \mathrm{CI})\end{array}$ & Totals not selected \\
\hline 6 Venous thromboembolism & 1 & & $\begin{array}{l}\text { Odds Ratio (M-H, } \\
\text { Fixed, } 95 \% \mathrm{CI})\end{array}$ & Totals not selected \\
\hline 7 Pulmonary embolus & 1 & & $\begin{array}{l}\text { Odds Ratio (M-H, } \\
\text { Fixed, } 95 \% \mathrm{CI})\end{array}$ & Totals not selected \\
\hline
\end{tabular}

Comparison 9

Combined therapy vs Placebo (in secondary prevention)

\begin{tabular}{|c|c|c|c|c|}
\hline Outcome or subgroup title & No. of studies & No. of participants & Statistical method & Effect size \\
\hline 1 Death (all causes) & 5 & 5772 & $\begin{array}{l}\text { Odds Ratio (M-H, } \\
\text { Fixed, 95\% CI) }\end{array}$ & $1.13[0.95,1.35]$ \\
\hline 2 Death (CV cause) & 5 & 5772 & $\begin{array}{l}\text { Odds Ratio (M-H, } \\
\text { Fixed, 95\% CI) }\end{array}$ & $1.11[0.87,1.42]$ \\
\hline $\begin{array}{l}3 \text { Non-fatal myocardial } \\
\text { infarction }\end{array}$ & 4 & 5712 & $\begin{array}{l}\text { Odds Ratio (M-H, } \\
\text { Fixed, 95\% CI) }\end{array}$ & $0.96[0.78,1.17]$ \\
\hline 4 Stroke & 5 & 5772 & $\begin{array}{l}\text { Odds Ratio (M-H, } \\
\text { Fixed, 95\% CI) }\end{array}$ & $1.16[0.94,1.44]$ \\
\hline 5 Combined CV events & 5 & 5772 & $\begin{array}{l}\text { Odds Ratio (M-H, } \\
\text { Fixed, 95\% CI) }\end{array}$ & $0.99[0.88,1.11]$ \\
\hline 6 Venous thromboembolism & 5 & 5852 & $\begin{array}{l}\text { Odds Ratio (M-H, } \\
\text { Fixed, 95\% CI) }\end{array}$ & $2.24[1.45,3.46]$ \\
\hline 7 Pulmonary embolus & 3 & 3224 & $\begin{array}{l}\text { Odds Ratio (M-H, } \\
\text { Fixed, } 95 \% \text { CI) }\end{array}$ & $2.88[1.21,6.82]$ \\
\hline
\end{tabular}

Analysis 1.1

Comparison 1 HRT vs placebo (in primary prevention), Outcome 1 Death (all causes)

Review: Hormone replacement therapy for preventing cardiovascular disease in postmenopausal women

Comparison: 1 HRT vs placebo (in primary prevention)

Outcome: 1 Death (all causes)

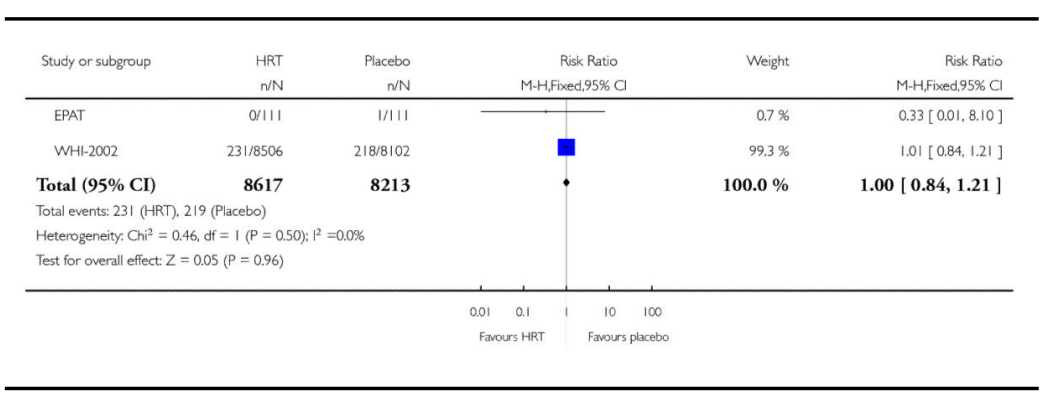


Analysis 1.2

Comparison 1 HRT vs placebo (in primary prevention), Outcome 2 Death (CV cause)

Review: Hormone replacement therapy for preventing cardiovascular disease in postmenopausal women

Comparison: 1 HRT vs placebo (in primary prevention)

Outcome: 2 Death (CV cause)

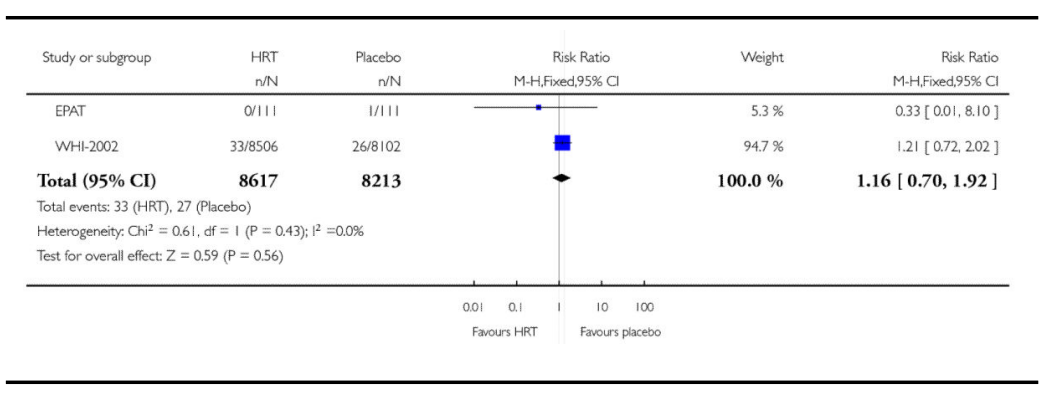

Analysis 1.3

Comparison 1 HRT vs placebo (in primary prevention), Outcome 3 Non-fatal MI

Review: Hormone replacement therapy for preventing cardiovascular disease in postmenopausal women

Comparison: 1 HRT vs placebo (in primary prevention)

Outcome: 3 Non-fatal MI

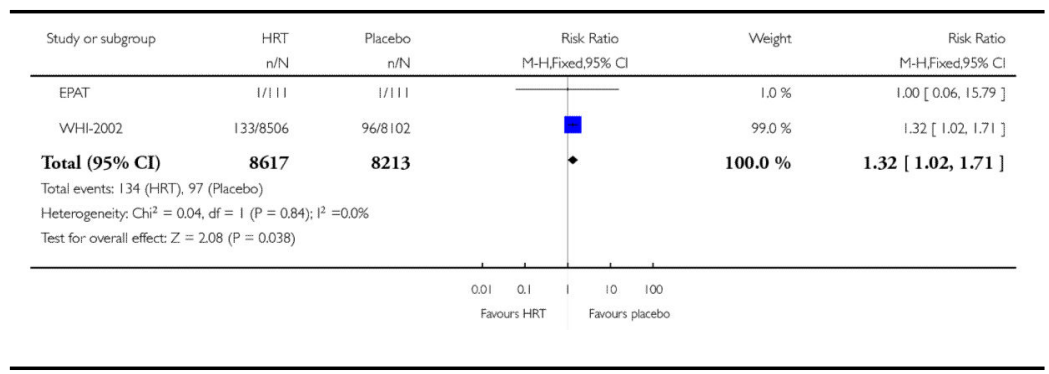


Analysis 1.4

Comparison 1 HRT vs placebo (in primary prevention), Outcome 4 Stroke

Review: Hormone replacement therapy for preventing cardiovascular disease in postmenopausal women

Comparison: 1 HRT vs placebo (in primary prevention)

Outcome: 4 Stroke

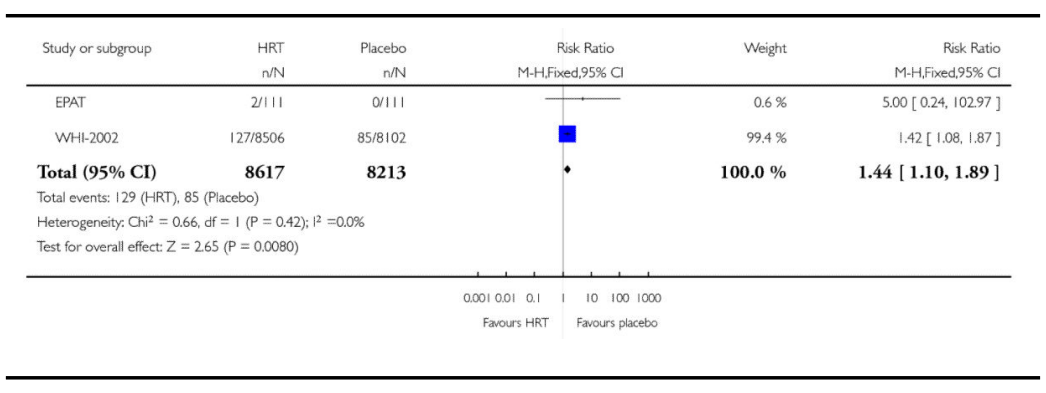

Analysis 1.5

Comparison 1 HRT vs placebo (in primary prevention), Outcome 5 Combined CV events and outcomes

Review: Hormone replacement therapy for preventing cardiovascular disease in postmenopausal women

Comparison: 1 HRT vs placebo (in primary prevention)

Outcome: 5 Combined CV events and outcomes

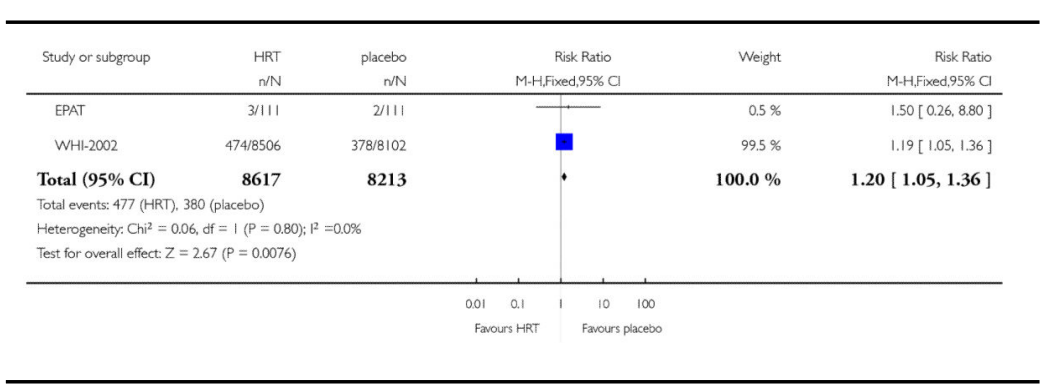




\section{Analysis 1.6}

Comparison 1 HRT vs placebo (in primary prevention), Outcome 6 Venous thromboembolism

Review: Hormone replacement therapy for preventing cardiovascular disease in postmenopausal women

Comparison: 1 HRT vs placebo (in primary prevention)

Outcome: 6 Venous thromboembolism

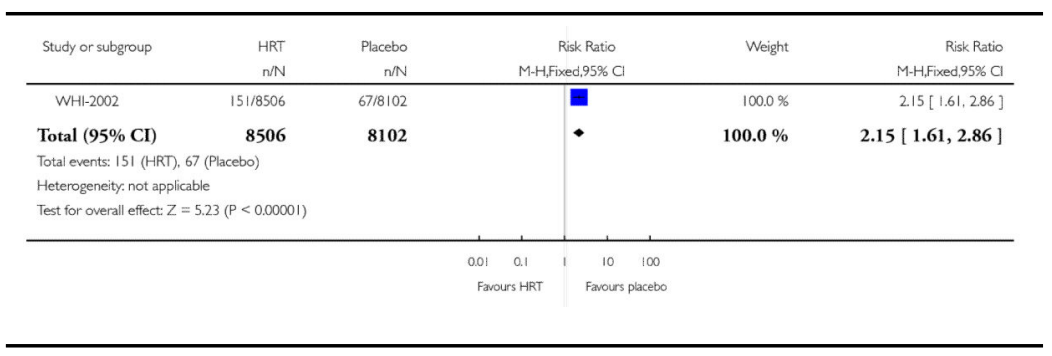

Analysis 1.7

Comparison 1 HRT vs placebo (in primary prevention), Outcome 7 Pulmonary embolus

Review: Hormone replacement therapy for preventing cardiovascular disease in postmenopausal women

Comparison: 1 HRT vs placebo (in primary prevention)

Outcome: 7 Pulmonary embolus

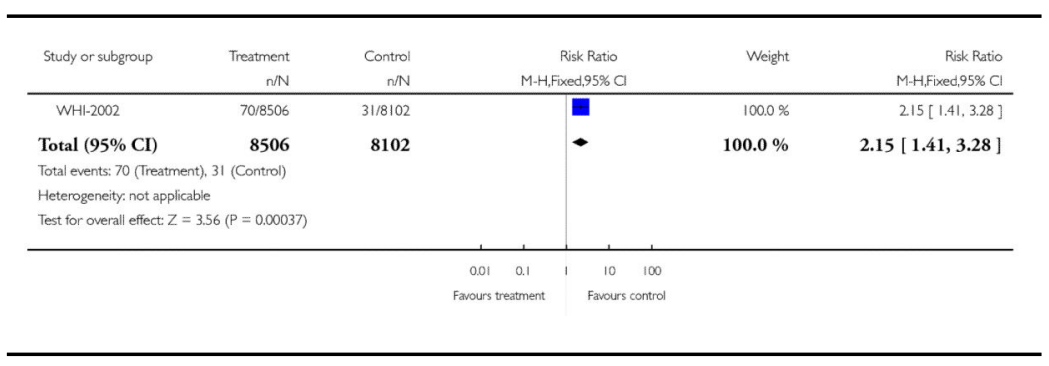


Analysis 2.1

\section{Comparison 2 HRT vs placebo (secondary prevention} only), Outcome 1 Death (all causes)

Review: Hormone replacement therapy for preventing cardiovascular disease in postmenopausal women

Comparison: 2 HRT vs placebo (secondary prevention only)

Outcome: 1 Death (all causes)

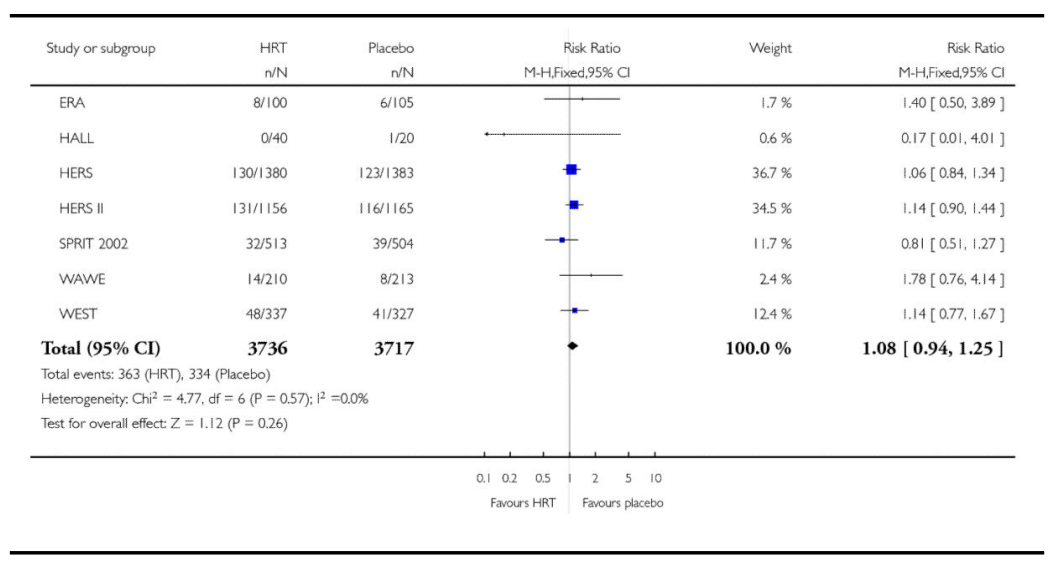

Analysis 2.2

Comparison 2 HRT vs placebo (secondary prevention only), Outcome 2 Death (CV cause)

Review: Hormone replacement therapy for preventing cardiovascular disease in postmenopausal women

Comparison: 2 HRT vs placebo (secondary prevention only)

Outcome: 2 Death (CV cause)

\begin{tabular}{|c|c|c|c|c|c|}
\hline Study or subgroup & $\begin{array}{l}\text { HRT } \\
\mathrm{n} N \mathrm{~N}\end{array}$ & $\begin{array}{r}\text { Placebo } \\
n / N\end{array}$ & $\begin{array}{c}\text { Risk Ratio } \\
\text { M-H,Fixed,95\% Cl }\end{array}$ & Weight & $\begin{array}{r}\text { Risk Ratio } \\
\text { M-H,Fixed,95\% C }\end{array}$ \\
\hline ERA & $4 / 100$ & $3 / 105$ & + & $1.7 \%$ & $1.40[0.32 .6 .10]$ \\
\hline HALL & $0 / 40$ & $1 / 20$ & & $1.1 \%$ & $0.17[0.01,4.01]$ \\
\hline HERS & $70 / 1380$ & $59 / 1383$ & $=$ & $33.5 \%$ & $1.19[0.85,1.67]$ \\
\hline HERS ॥ & $62 / 1156$ & $63 / 1165$ & $=$ & $35.7 \%$ & $0.99[0.71,1.39]$ \\
\hline SPRIT 2002 & $21 / 513$ & $30 / 504$ & $\rightarrow$ & $17.2 \%$ & $0.69[0.40,1.18]$ \\
\hline WAWE & $8 / 210$ & $6 / 213$ & $\longrightarrow$ & $3.4 \%$ & $1.35[0.48,3.83]$ \\
\hline WEST & |1/337 & $13 / 327$ & $\longrightarrow$ & $7.5 \%$ & $0.82[0.37,1.81]$ \\
\hline Total $(95 \%$ CI) & 3736 & 3717 & - & $100.0 \%$ & $1.00[0.82,1.23]$ \\
\hline \multirow{3}{*}{\multicolumn{6}{|c|}{$\begin{array}{l}\text { Total events: } 176(\mathrm{HRT}), 175(\mathrm{Placebo}) \\
\text { Heterogeneity: Chi }=4.79, \mathrm{df}=6(\mathrm{P}=0.57) ; 1^{2}=0.0 \% \\
\text { Test for overal effect: } Z=0.02(P=0.98)\end{array}$}} \\
\hline & & & & & \\
\hline & & & & & \\
\hline & & & 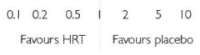 & & \\
\hline
\end{tabular}


Analysis 2.3

Comparison 2 HRT vs placebo (secondary prevention only), Outcome 3 Non-fatal MI

Review: Hormone replacement therapy for preventing cardiovascular disease in postmenopausal women

Comparison: 2 HRT vs placebo (secondary prevention only)

Outcome: 3 Non-fatal MI

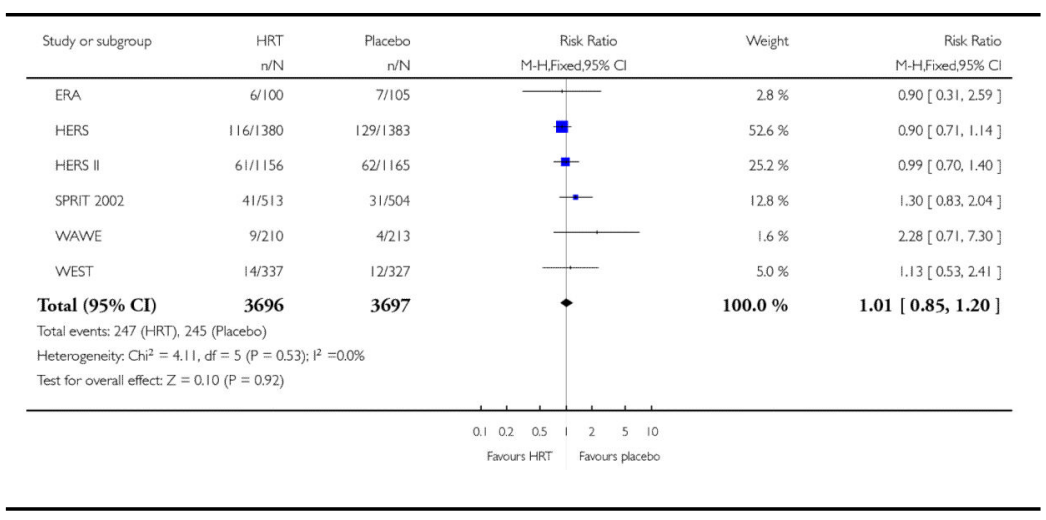

Analysis 2.4

Comparison 2 HRT vs placebo (secondary prevention only), Outcome 4 Stroke

Review: Hormone replacement therapy for preventing cardiovascular disease in postmenopausal women

Comparison: 2 HRT vs placebo (secondary prevention only)

Outcome: 4 Stroke

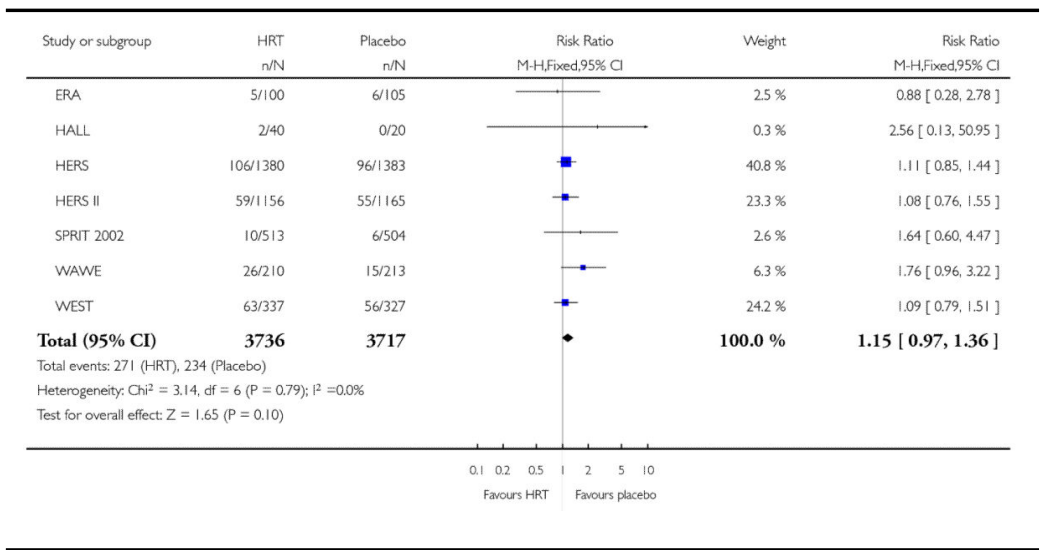


Analysis 2.5

Comparison 2 HRT vs placebo (secondary prevention only), Outcome 5 Combined CV events and outcomes

Review: Hormone replacement therapy for preventing cardiovascular disease in postmenopausal women

Comparison: 2 HRT vs placebo (secondary prevention only)

Outcome: 5 Combined CV events and outcomes

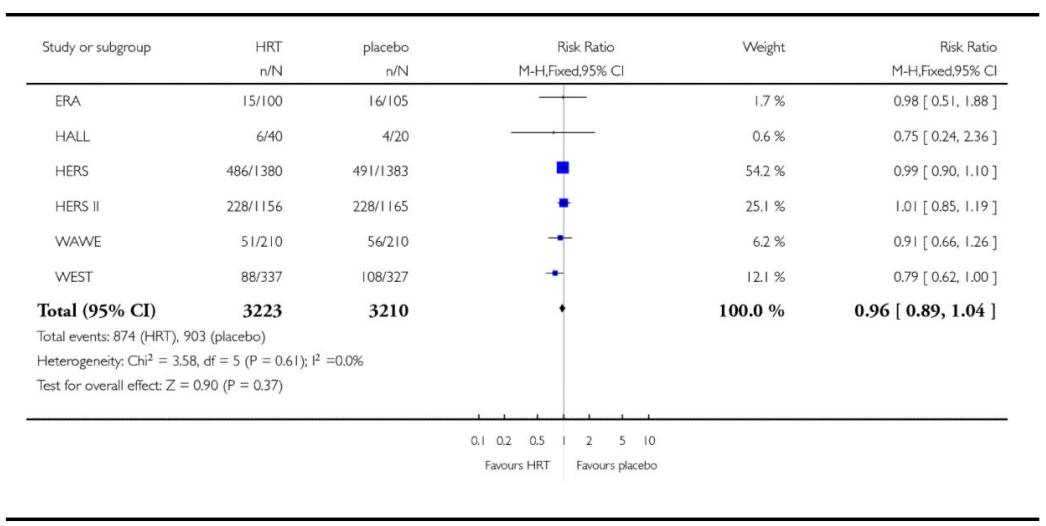

Analysis 2.6

Comparison 2 HRT vs placebo (secondary prevention only), Outcome 6 Venous thromboembolism

Review: Hormone replacement therapy for preventing cardiovascular disease in postmenopausal women

Comparison: 2 HRT vs placebo (secondary prevention only)

Outcome: 6 Venous thromboembolism

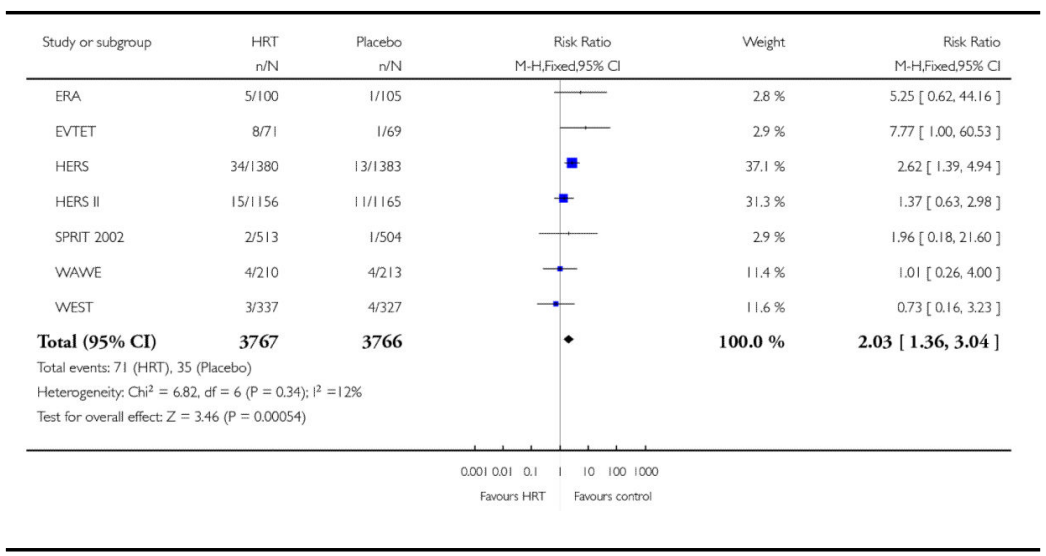


Analysis 2.7

\section{Comparison 2 HRT vs placebo (secondary prevention} only), Outcome 7 Pulmonary embolus

Review: Hormone replacement therapy for preventing cardiovascular disease in postmenopausal women

Comparison: 2 HRT vs placebo (secondary prevention only)

Outcome: 7 Pulmonary embolus

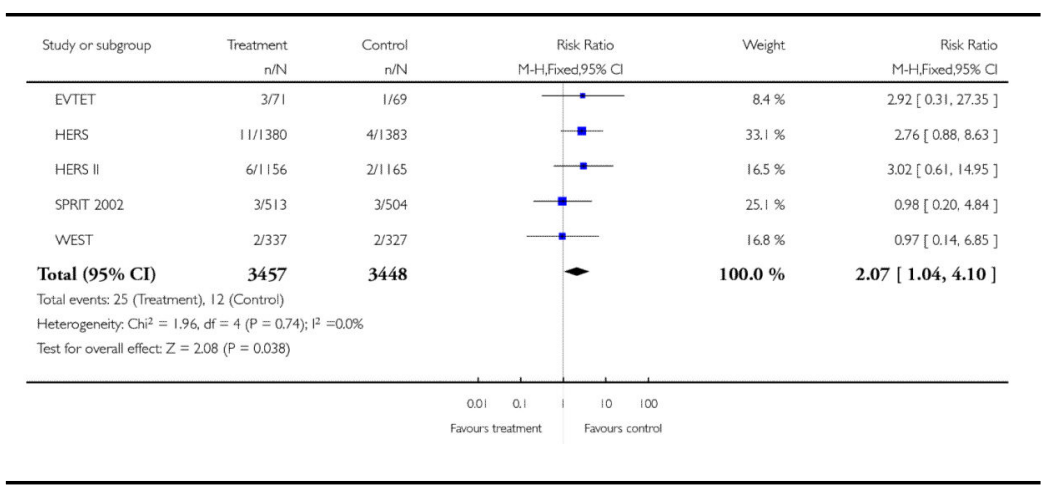

\section{Analysis 3.1}

Comparison 3 HRT vs placebo (in primary or secondary prevention), Outcome 1 Death (all causes)

Review: Hormone replacement therapy for preventing cardiovascular disease in postmenopausal women Comparison: 3 HRT vs placebo (in primary or secondary prevention) Outcome: 1 Death (all causes)

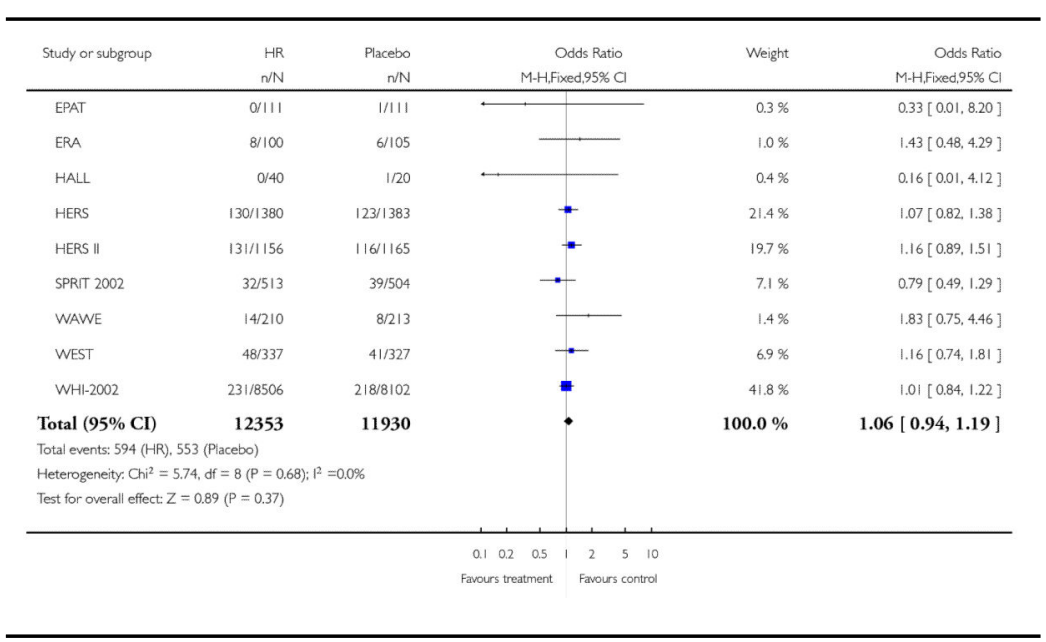


Analysis 3.2

Comparison 3 HRT vs placebo (in primary or secondary prevention), Outcome 2 Death (CV cause)

Review: Hormone replacement therapy for preventing cardiovascular disease in postmenopausal women

Comparison: 3 HRT vs placebo (in primary or secondary prevention)

Outcome: 2 Death (CV cause)

\begin{tabular}{|c|c|c|c|c|c|}
\hline Study or subgroup & $\begin{array}{l}\mathrm{HRT} \\
\mathrm{NN}\end{array}$ & $\begin{array}{r}\text { Placebo } \\
\text { niN }\end{array}$ & $\begin{array}{r}\text { Odds Ratio } \\
\text { M-H,Fixed,95\% Cl }\end{array}$ & Weight & $\begin{array}{r}\text { Odds Ratio } \\
\text { M-H,Fixed,95\% CI }\end{array}$ \\
\hline ЕРAT & $0 / 111$ & $1 / 111$ & & $0.8 \%$ & $0.33[0.01,8.20]$ \\
\hline ERA & $4 / 100$ & 3/105 & + & $1.4 \%$ & $1.42[0.31,6.49]$ \\
\hline HALL & $1 / 40$ & $0 / 20$ & & $0.3 \%$ & $1.56[0.06,39.95]$ \\
\hline HERS & $70 / 1380$ & $59 / 1383$ & - & $28.8 \%$ & $1.20[0.84,1.71]$ \\
\hline HERS $\|$ & $62 / 1156$ & $63 / 1165$ & $\rightarrow$ & $30.6 \%$ & $0.99[0.69,1.42]$ \\
\hline SPRIT 2002 & $21 / 513$ & $30 / 504$ & $\because$ & $14.9 \%$ & $0.67[0.38,1.19]$ \\
\hline WAWE & $8 / 210$ & $6 / 213$ & $\longrightarrow$ & $29 \%$ & $1.37[0.47,4.01]$ \\
\hline WEST & $11 / 337$ & $13 / 327$ & $\longrightarrow$ & $6.6 \%$ & $0.82[0.36,1.85]$ \\
\hline$W H 1-2002$ & $33 / 8506$ & $26 / 8102$ & - & $13.7 \%$ & $1.21[0.72,2.02]$ \\
\hline Total $(95 \% \mathrm{CI})$ & 12353 & 11930 & - & $100.0 \%$ & $1.04[0.85,1.26]$ \\
\hline \multicolumn{6}{|c|}{$\begin{array}{l}\text { Total events: } 210 \text { (HRT), } 201 \text { (Placebo) } \\
\text { Heterogeneity: Chi }{ }^{2}=4.52, \mathrm{df}=8(\mathrm{P}=0.81) ; \mathrm{I}^{2}=0.0 \% \\
\text { Test for overall effect: } Z=0.35(P=0.72)\end{array}$} \\
\hline & & & 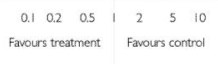 & & \\
\hline
\end{tabular}

Analysis 3.3

Comparison 3 HRT vs placebo (in primary or secondary prevention), Outcome 3 Non-fatal MI

Review: Hormone replacement therapy for preventing cardiovascular disease in postmenopausal women

Comparison: 3 HRT vs placebo (in primary or secondary prevention) Outcome: 3 Non-fatal MI

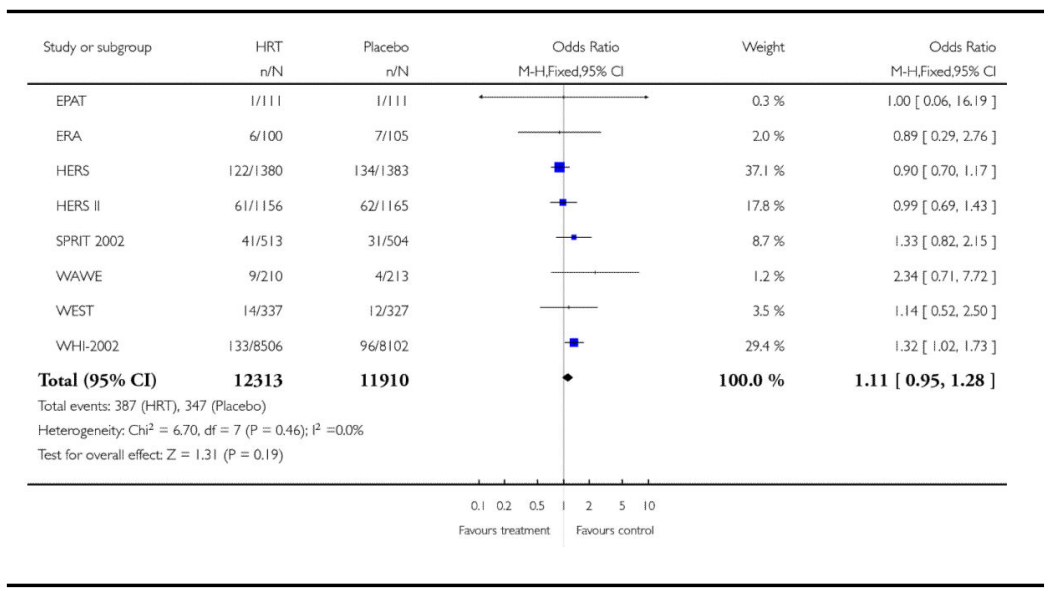


Analysis 3.4

\section{Comparison 3 HRT vs placebo (in primary or} secondary prevention), Outcome 4 Stroke

Review: Hormone replacement therapy for preventing cardiovascular disease in postmenopausal women

Comparison: 3 HRT vs placebo (in primary or secondary prevention)

Outcome: 4 Stroke

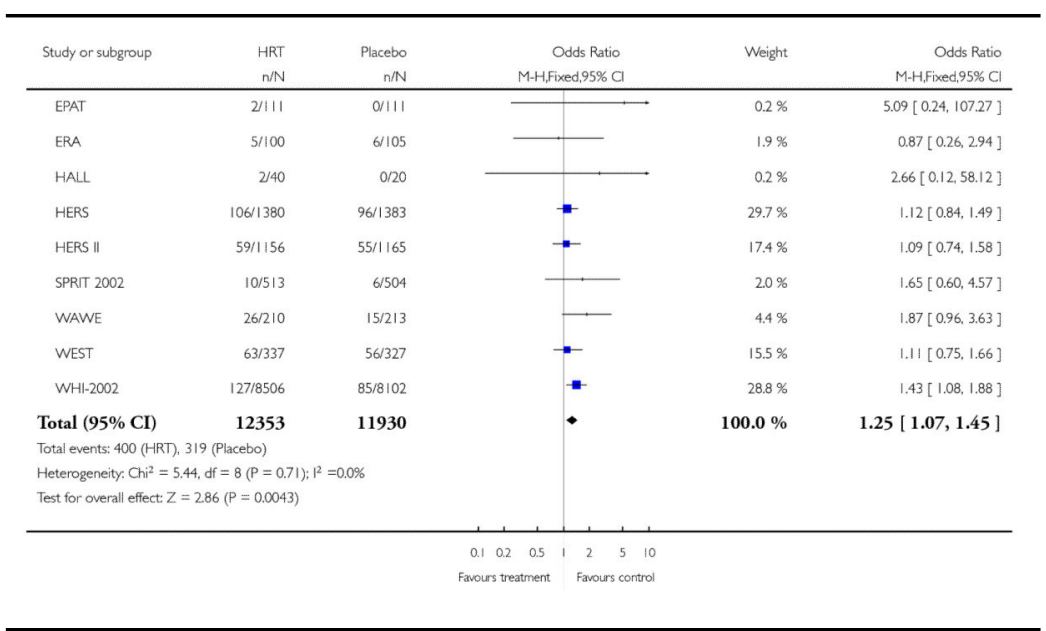

Analysis 3.5

Comparison 3 HRT vs placebo (in primary or secondary prevention), Outcome 5 Combined CV events and outcomes

Review: Hormone replacement therapy for preventing cardiovascular disease in postmenopausal women Comparison: 3 HRT vs placebo (in primary or secondary prevention) Outcome: 5 Combined CV events and outcomes

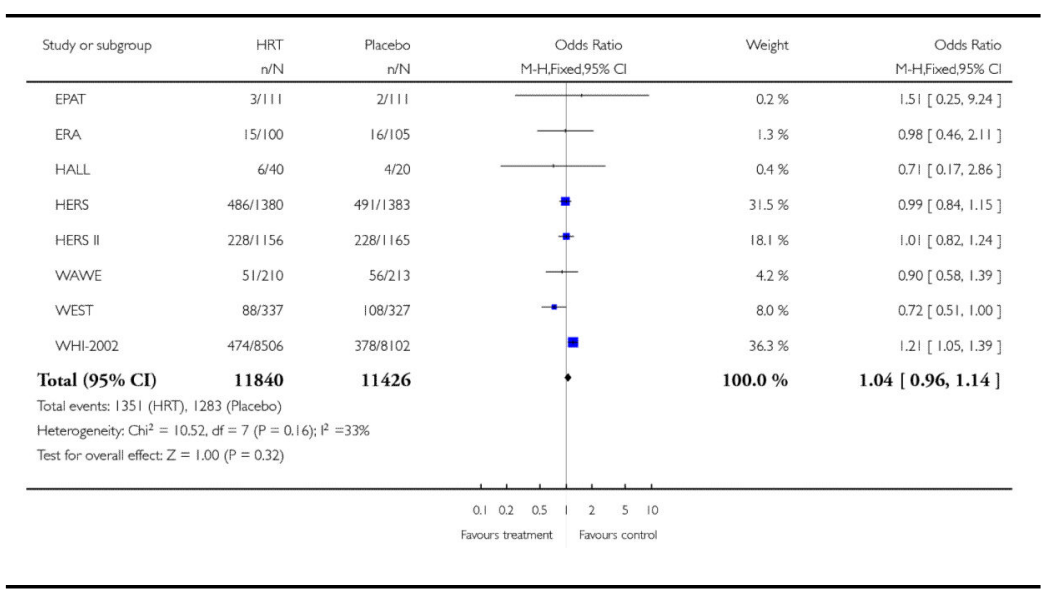


Analysis 3.6

Comparison 3 HRT vs placebo (in primary or secondary prevention), Outcome 6 Venous thromboembolism

Review: Hormone replacement therapy for preventing cardiovascular disease in postmenopausal women

Comparison: 3 HRT vs placebo (in primary or secondary prevention)

Outcome: 6 Venous thromboembolism

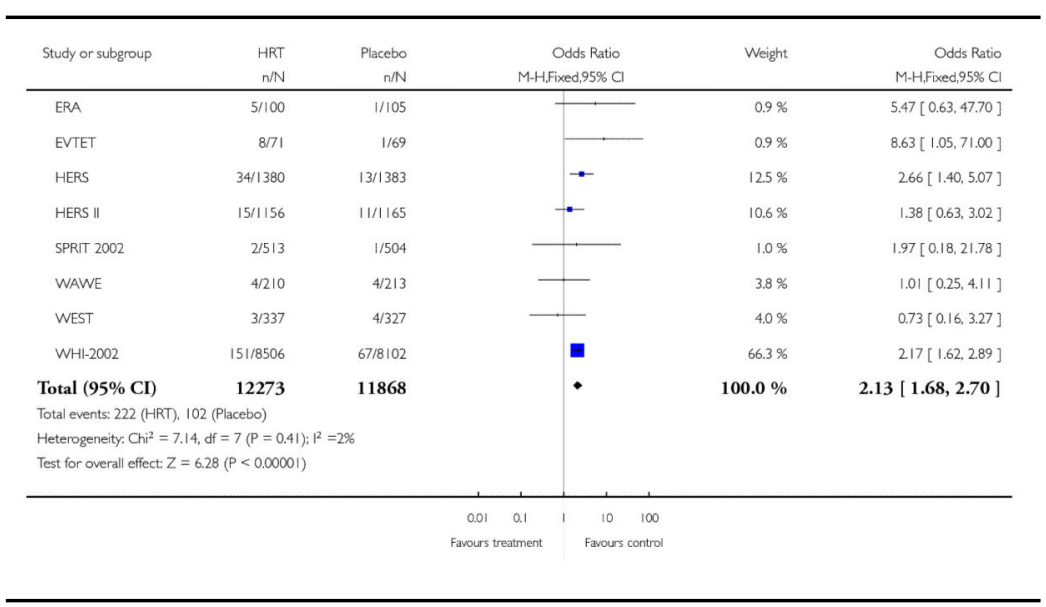

Analysis 3.7

Comparison 3 HRT vs placebo (in primary or secondary prevention), Outcome 7 Pulmonary embolus

Review: Hormone replacement therapy for preventing cardiovascular disease in postmenopausal women

Comparison: 3 HRT vs placebo (in primary or secondary prevention)

Outcome: 7 Pulmonary embolus

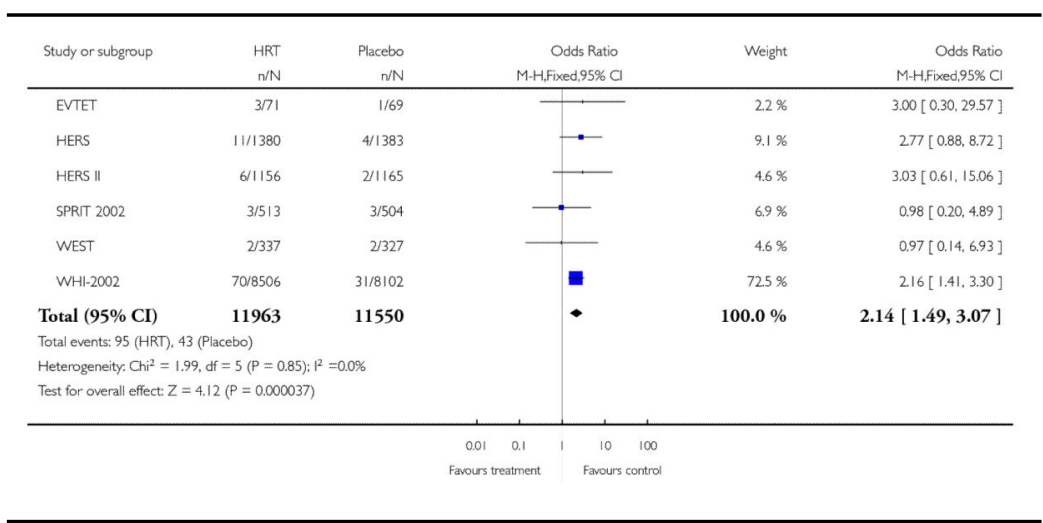




\section{Analysis 4.1}

Comparison 4 Oestrogen vs placebo (in primary and secondary prevention), Outcome 1 Death (all causes)

Review: Hormone replacement therapy for preventing cardiovascular disease in postmenopausal women

Comparison: 4 Oestrogen vs placebo (in primary and secondary prevention)

Outcome: 1 Death (all causes)

\begin{tabular}{|c|c|c|c|c|c|}
\hline Study or subgroup & $\begin{array}{r}\text { Oestrogen } \\
n / N\end{array}$ & $\begin{array}{r}\text { Placebo } \\
n / N\end{array}$ & $\begin{array}{r}\text { Odds Ratio } \\
\text { M-H,Fixed,95\% Cl }\end{array}$ & Weight & $\begin{array}{r}\text { Odds Ratio } \\
\text { M-H,Fixed,95\% CI }\end{array}$ \\
\hline EPAT & o/111 & $|1 / 1|$ & 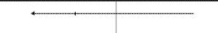 & $20 \%$ & $0.33[0.01,8.20]$ \\
\hline SPRIT 2002 & $32 / 513$ & 39/504 & $\rightarrow$ & $49.8 \%$ & $0.79[0.49,1.29]$ \\
\hline WEST & $48 / 337$ & 4/323 & $=$ & $48.2 \%$ & $1.16[0.74,1.81]$ \\
\hline Total $(95 \% \mathrm{CI})$ & 961 & 942 & - & $100.0 \%$ & $0.96[0.69,1.33]$ \\
\hline \multicolumn{6}{|c|}{$\begin{array}{l}\text { Total events: } 80 \text { (Oestrogen). } 81 \text { (Placebo) } \\
\text { Heterogeneity: Chi }=1.70, \text { ff }=2(P=0.43) ; 1^{2}=0.0 \% \\
\text { Test for overall effect: } Z=0.25(P=0.81)\end{array}$} \\
\hline & & & 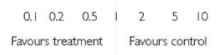 & & \\
\hline
\end{tabular}

Analysis 4.2

Comparison 4 Oestrogen vs placebo (in primary and secondary prevention), Outcome 2 Death (CV causes)

Review: Hormone replacement therapy for preventing cardiovascular disease in postmenopausal women

Comparison: 4 Oestrogen vs placebo (in primary and secondary prevention) Outcome: 2 Death (CV causes)

\begin{tabular}{|c|c|c|c|c|c|}
\hline Study or subgroup & $\begin{array}{r}\text { Oestrogen } \\
\mathrm{n} / \mathrm{N}\end{array}$ & $\begin{array}{r}\text { Placebo } \\
n \mathbb{N}\end{array}$ & $\begin{array}{r}\text { Odds Ratio } \\
\text { M-H.Fixed,95\% C }\end{array}$ & Weight & $\begin{array}{r}\text { Odds Ratio } \\
\text { M-H,Fixed,95\% C }\end{array}$ \\
\hline EPAT & $0 / 111$ & $1 / 111$ & - & $3.4 \%$ & $0.33[0.01,8.20]$ \\
\hline SPRTT 2002 & $21 / 513$ & 30/504 & $=$ & $67.1 \%$ & $0.67[0.38,1.19]$ \\
\hline WEST & 11/337 & 13/327 & 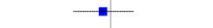 & $29.5 \%$ & $0.82[0.36,1.85]$ \\
\hline Total $(95 \% \mathrm{CI})$ & 961 & 942 & - & $100.0 \%$ & $0.70[0.44,1.12]$ \\
\hline \multirow{2}{*}{\multicolumn{6}{|c|}{$\begin{array}{l}\text { Total events: } 32 \text { (Oestrogen), } 44 \text { (Placebo) } \\
\text { Heterogeneity: Chi }{ }^{2}=0.36, \text { df }=2(P=0.84): 1^{2}=0.0 \%\end{array}$}} \\
\hline & & & & & \\
\hline \multicolumn{6}{|c|}{ Test for overal effect: $Z=1.49(P=0.14)$} \\
\hline & & & 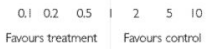 & & \\
\hline
\end{tabular}




\section{Analysis 4.3}

Comparison 4 Oestrogen vs placebo (in primary and secondary prevention), Outcome 3 Nonfatal MI

Review: Hormone replacement therapy for preventing cardiovascular disease in postmenopausal women

Comparison: 4 Oestrogen vs placebo (in primary and secondary prevention)

Outcome: 3 Non-fatal MI

\begin{tabular}{|c|c|c|c|c|c|}
\hline Study or subgroup & $\begin{array}{r}\text { Oestrogen } \\
n / N\end{array}$ & $\begin{array}{r}\text { Placebo } \\
\mathrm{n} / \mathrm{N}\end{array}$ & $\begin{array}{r}\text { Odds Ratio } \\
\text { M-H,Fixed,95\% Cl }\end{array}$ & Weight & $\begin{array}{r}\text { Odds Ratio } \\
\text { M-H,Fixed,95\% Cl }\end{array}$ \\
\hline EPAT & 1/111 & $1 / 111$ & $\longrightarrow$ & $2.4 \%$ & $1.00[0.06,16.19]$ \\
\hline SPRT 2002 & $41 / 513$ & $31 / 504$ & $=$ & $69.4 \%$ & $1.33[0.82,2.15]$ \\
\hline WEST & 14/337 & $12 / 327$ & $\longrightarrow$ & $28.2 \%$ & $1.14[0.52,2.50]$ \\
\hline Total $(95 \% \mathrm{CI})$ & 961 & 942 & - & $100.0 \%$ & $1.26[0.84,1.90]$ \\
\hline \multirow{2}{*}{\multicolumn{6}{|c|}{$\begin{array}{l}\text { Total events: } 56 \text { (Oestrogen), } 44 \text { (Placebo) } \\
\text { Heterogeneity, } \mathrm{Ch}^{2}=0.13, \mathrm{df}=2(\mathrm{P}=0.94): 1^{2}=0.0 \%\end{array}$}} \\
\hline & & & & & \\
\hline \multicolumn{6}{|c|}{ Test for overall effect: $Z=1.13(P=0.26)$} \\
\hline & & & $\begin{array}{cccccc}0.1 & 0.2 & 0.5 & 2 & 5 & 10 \\
\text { Farours treatment } & \text { Favours control }\end{array}$ & & \\
\hline
\end{tabular}

Analysis 4.4

Comparison 4 Oestrogen vs placebo (in primary and secondary prevention), Outcome 4 Stroke

Review: Hormone replacement therapy for preventing cardiovascular disease in postmenopausal women

Comparison: 4 Oestrogen vs placebo (in primary and secondary prevention)

Outcome: 4 Stroke

\begin{tabular}{|c|c|c|c|c|c|}
\hline Study or subgroup & $\begin{array}{r}\text { Destrogen } \\
n / N\end{array}$ & $\begin{array}{r}\text { Placebo } \\
\mathrm{n} / \mathrm{N}\end{array}$ & $\begin{array}{l}\text { Odds Ratio } \\
\text { M-H,Fixed,95\% Cl }\end{array}$ & Weight & $\begin{array}{r}\text { Odds Ratio } \\
\text { M-H,Fixed,95\% CI }\end{array}$ \\
\hline EPAT & $2 / 111$ & $0 / 111$ & & $0.9 \%$ & $5.09[0.24,107.27]$ \\
\hline SPRIT 2002 & $10 / 513$ & 6/504 & 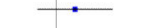 & $11.3 \%$ & $1.65[0.60,4.57]$ \\
\hline WEST & $63 / 337$ & $56 / 327$ & $=$ & $87.8 \%$ & $1.11[0.75,1.66]$ \\
\hline Total $(95 \% \mathrm{CI})$ & 961 & 942 & 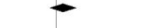 & $100.0 \%$ & $1.21[0.84,1.74]$ \\
\hline \multirow{2}{*}{\multicolumn{6}{|c|}{$\begin{array}{l}\text { Total events: } 75 \text { (Oestrogen), } 62 \text { (Placebo) } \\
\text { Heterogeneity: Chi }{ }^{2}=1.38, \mathrm{df}=2(\mathrm{P}=0.50) ; 1^{2}=0.0 \%\end{array}$}} \\
\hline & & & & & \\
\hline \multicolumn{6}{|c|}{ Test for overal effect: $Z=1.02(P=0.31)$} \\
\hline & & & $\begin{array}{cccc}0.5 & 2 & 5 & 10 \\
\text { eatment } & \text { Favours control }\end{array}$ & & \\
\hline
\end{tabular}




\section{Analysis 4.5}

Comparison 4 Oestrogen vs placebo (in primary and secondary prevention), Outcome 5 Combined CV events and outcomes

Review: Hormone replacement therapy for preventing cardiovascular disease in postmenopausal women

Comparison: 4 Oestrogen vs placebo (in primary and secondary prevention)

Outcome: 5 Combined CV events and outcomes

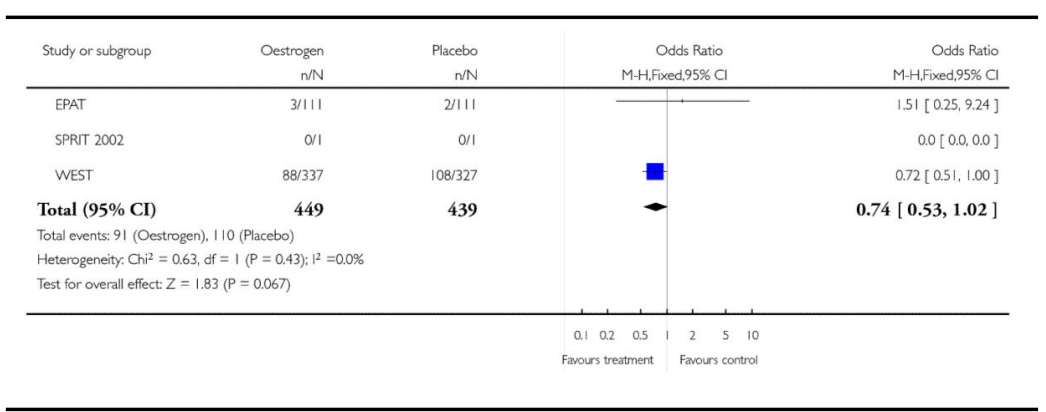

Analysis 4.6

Comparison 4 Oestrogen vs placebo (in primary and secondary prevention), Outcome 6 Venous thromboembolism

Review: Hormone replacement therapy for preventing cardiovascular disease in postmenopausal women

Comparison: 4 Oestrogen vs placebo (in primary and secondary prevention) Outcome: 6 Venous thromboembolism

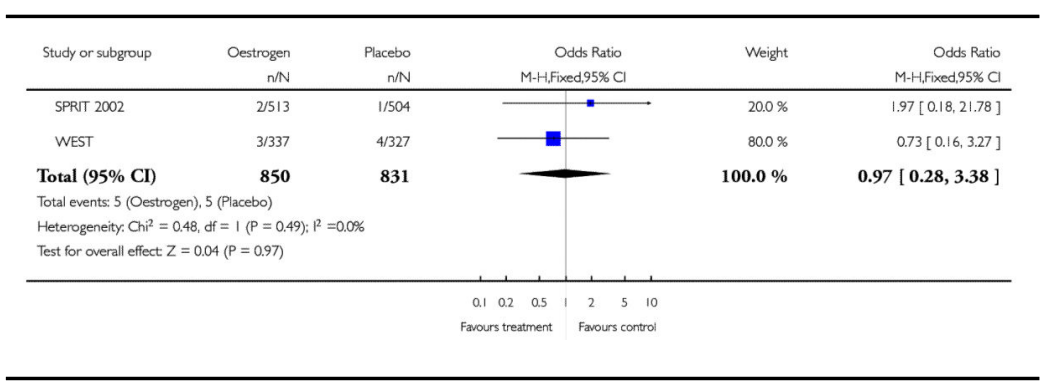




\section{Analysis 4.7}

Comparison 4 Oestrogen vs placebo (in primary and secondary prevention), Outcome 7 Pulmonary embolus

Review: Hormone replacement therapy for preventing cardiovascular disease in postmenopausal women

Comparison: 4 Oestrogen vs placebo (in primary and secondary prevention)

Outcome: 7 Pulmonary embolus

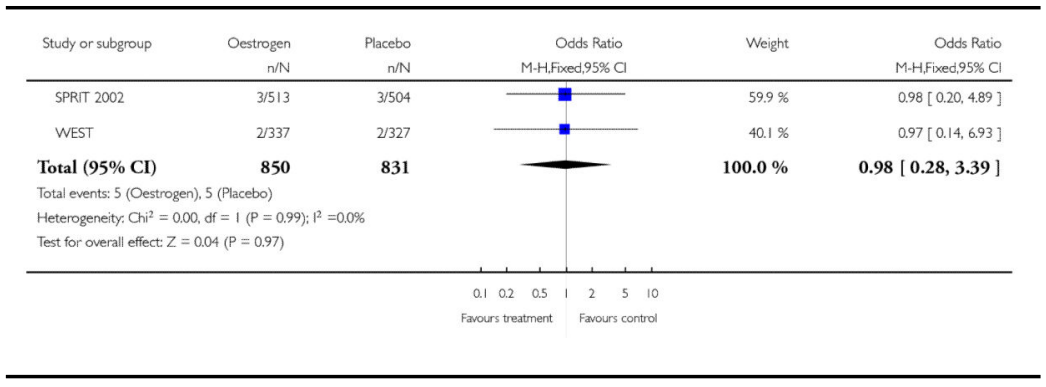

\section{Analysis 5.1}

Comparison 5 Oestrogen vs placebo (in primary prevention), Outcome 1 Death (all causes)

Review: Hormone replacement therapy for preventing cardiovascular disease in postmenopausal women

Comparison: 5 Oestrogen vs placebo (in primary prevention) Outcome: 1 Death (all causes)

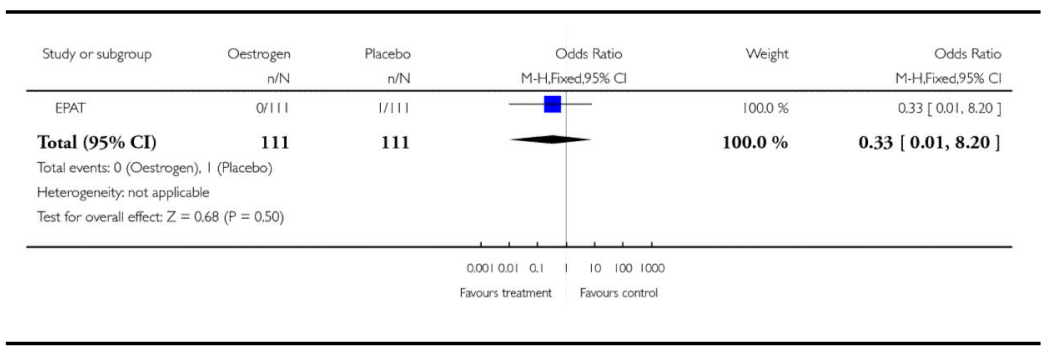


Analysis 5.2

Comparison 5 Oestrogen vs placebo (in primary prevention), Outcome 2 Death ( $\mathrm{CV}$ cause)

Review: Hormone replacement therapy for preventing cardiovascular disease in postmenopausal women

Comparison: 5 Oestrogen vs placebo (in primary prevention)

Outcome: 2 Death (CV cause)

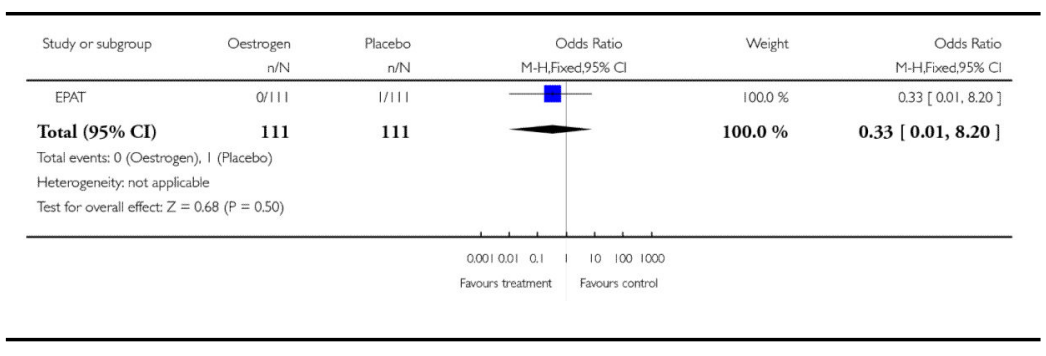

Analysis 5.3

Comparison 5 Oestrogen vs placebo (in primary prevention), Outcome 3 Non-fatal MI

Review: Hormone replacement therapy for preventing cardiovascular disease in postmenopausal women

Comparison: 5 Oestrogen vs placebo (in primary prevention)

Outcome: 3 Non-fatal MI

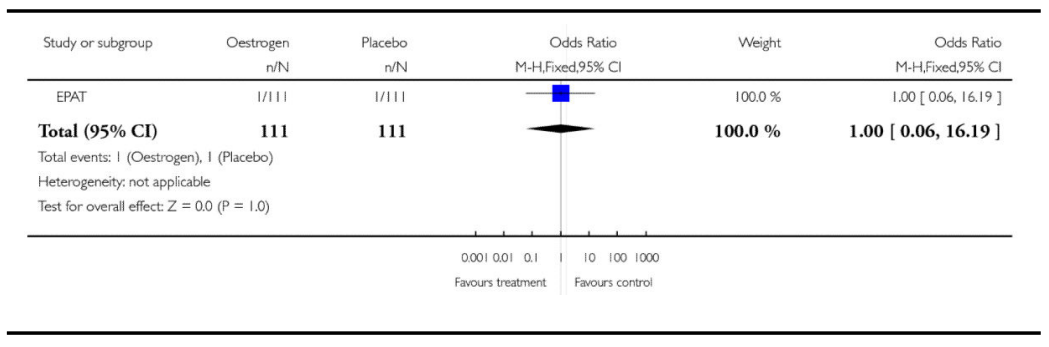


Analysis 5.4

Comparison 5 Oestrogen vs placebo (in primary prevention), Outcome 4 Stroke

Review: Hormone replacement therapy for preventing cardiovascular disease in postmenopausal women

Comparison: 5 Oestrogen vs placebo (in primary prevention)

Outcome: 4 Stroke

\begin{tabular}{|c|c|c|c|c|c|}
\hline Study or subgroup & $\begin{array}{r}\text { Oestrogen } \\
n / N\end{array}$ & $\begin{array}{r}\text { Placebo } \\
\text { n/N }\end{array}$ & $\begin{array}{l}\text { Odds Ratio } \\
\text { M-H,Fixed, } 95 \% \text { Cl }\end{array}$ & Weight & $\begin{array}{r}\text { Odds Ratio } \\
\text { M-H,Fixed.95\% CI }\end{array}$ \\
\hline EPAT & $2 / 111$ & $0 / 111$ & 回 & $100.0 \%$ & $5.09[0.24,107.27]$ \\
\hline \multicolumn{6}{|c|}{$\begin{array}{l}\text { Total events: } 2 \text { (Oestrogen), } 0 \text { (Placebo) } \\
\text { Heterogeneity, not applicable } \\
\text { Test for overal leffect: } Z=1.05(P=0.30)\end{array}$} \\
\hline & & & \begin{tabular}{c|ccc}
$0.00 \mid 0.01$ & 0.1 \\
Favours treatment & 10 & 100 & 1000 \\
Farours control
\end{tabular} & & \\
\hline
\end{tabular}

Analysis 5.5

Comparison 5 Oestrogen vs placebo (in primary prevention), Outcome 5 Combined $\mathrm{CV}$ events

Review: Hormone replacement therapy for preventing cardiovascular disease in postmenopausal women

Comparison: 5 Oestrogen vs placebo (in primary prevention) Outcome: 5 Combined CV events

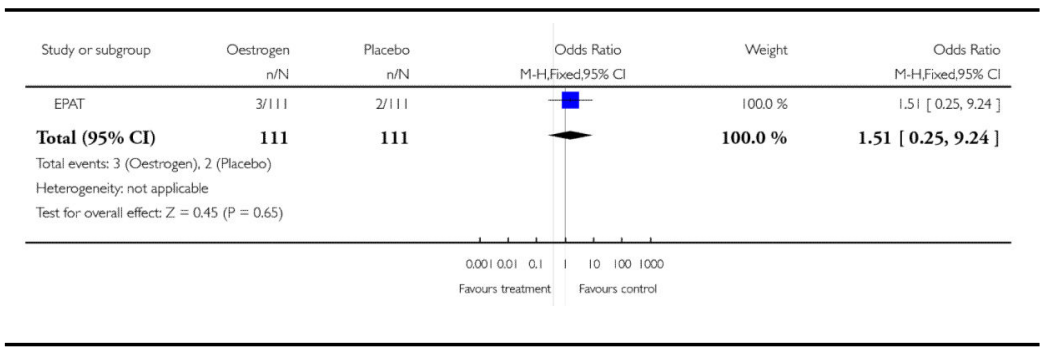


Analysis 6.1

Comparison 6 Oestrogen vs placebo (in secondary prevention), Outcome 1 Death (all causes)

Review: Hormone replacement therapy for preventing cardiovascular disease in postmenopausal women

Comparison: 6 Oestrogen vs placebo (in secondary prevention)

Outcome: 1 Death (all causes)

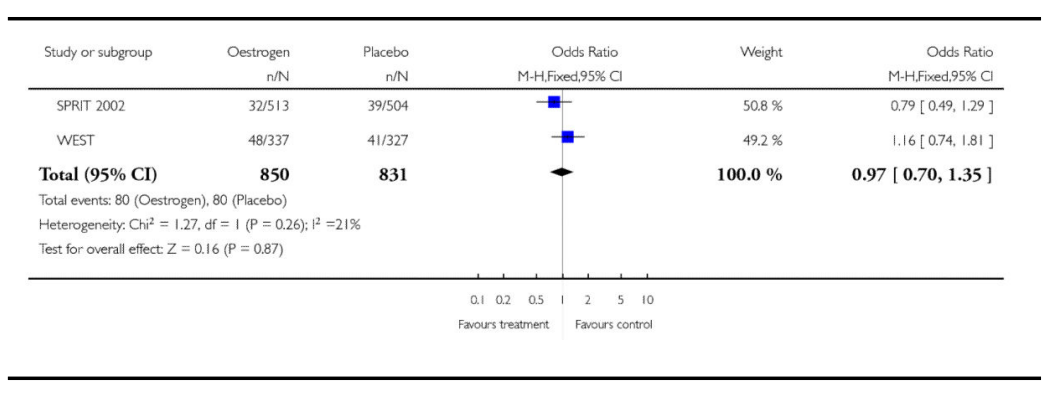

Analysis 6.2

Comparison 6 Oestrogen vs placebo (in secondary prevention), Outcome 2 Death (CV causes)

Review: Hormone replacement therapy for preventing cardiovascular disease in postmenopausal women

Comparison: 6 Oestrogen vs placebo (in secondary prevention)

Outcome: 2 Death (CV causes)

\begin{tabular}{|c|c|c|c|c|c|}
\hline Study or subgroup & $\begin{array}{r}\text { Oestrogen } \\
n / N\end{array}$ & $\begin{array}{r}\text { Placebo } \\
n / N\end{array}$ & $\begin{array}{r}\text { Odds Ratio } \\
\text { M-H,Fixed,95\% Cl }\end{array}$ & Weight & $\begin{array}{r}\text { Odds Ratio } \\
\text { M-H,Fixed,95\% Cl }\end{array}$ \\
\hline SPRT 2002 & $21 / 513$ & $30 / 504$ & $\rightarrow$ & $69.5 \%$ & $0.67[0.38,1.19]$ \\
\hline WEST & $11 / 337$ & $13 / 327$ & $\rightarrow$ & $30.5 \%$ & $0.82[0.36,1.85]$ \\
\hline Total $(95 \% \mathrm{CI})$ & 850 & 831 & - & $100.0 \%$ & $0.72[0.45,1.15]$ \\
\hline \multirow{3}{*}{\multicolumn{6}{|c|}{$\begin{array}{l}\text { Total events: } 32 \text { (Oestrogen), } 43 \text { (Placebo) } \\
\text { Heterogenety: Chi' }=0.14, \text { of }=1(P=0.71) ; 1^{2}=0.0 \% \\
\text { Test for overall effect: } Z=1.39(P=0.16)\end{array}$}} \\
\hline & & & & & \\
\hline & & & & & \\
\hline & & & 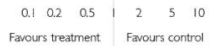 & & \\
\hline
\end{tabular}


Analysis 6.3

Comparison 6 Oestrogen vs placebo (in secondary prevention), Outcome 3 Non-fatal MI

Review: Hormone replacement therapy for preventing cardiovascular disease in postmenopausal women

Comparison: 6 Oestrogen vs placebo (in secondary prevention)

Outcome: 3 Non-fatal MI

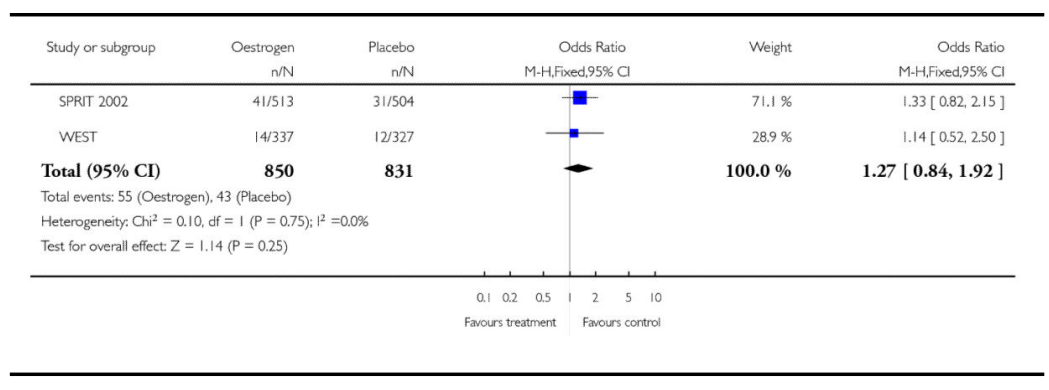

Analysis 6.4

Comparison 6 Oestrogen vs placebo (in secondary prevention), Outcome 4 Stroke

Review: Hormone replacement therapy for preventing cardiovascular disease in postmenopausal women

Comparison: 6 Oestrogen vs placebo (in secondary prevention)

Outcome: 4 Stroke

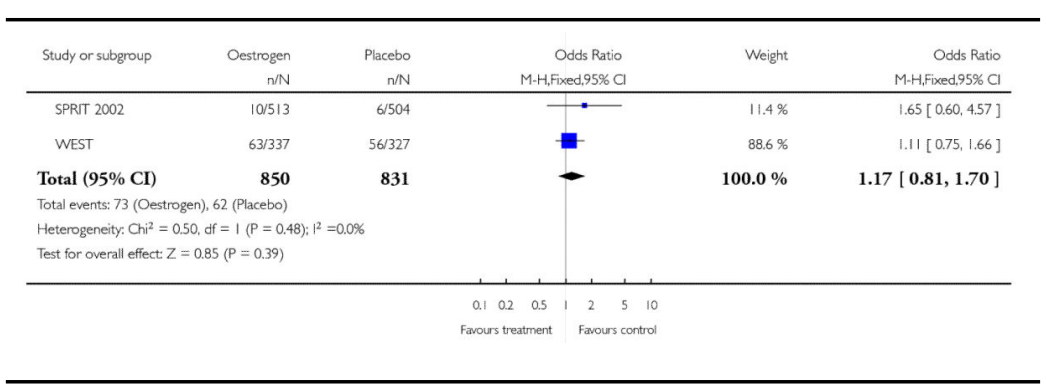




\section{Analysis 6.5}

Comparison 6 Oestrogen vs placebo (in secondary prevention), Outcome 5 Combined CV events and outcomes

Review: Hormone replacement therapy for preventing cardiovascular disease in postmenopausal women

Comparison: 6 Oestrogen vs placebo (in secondary prevention)

Outcome: 5 Combined CV events and outcomes

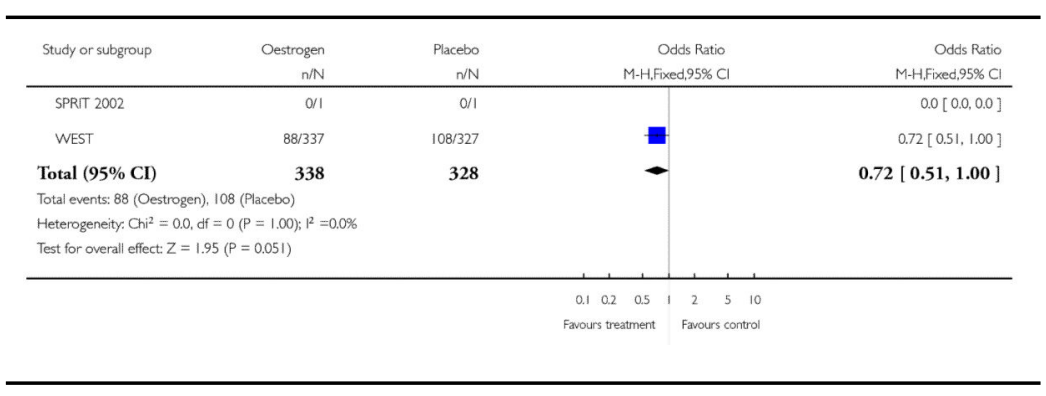

Analysis 6.6

Comparison 6 Oestrogen vs placebo (in secondary prevention), Outcome 6 Venous thromboembolism

Review: Hormone replacement therapy for preventing cardiovascular disease in postmenopausal women

Comparison: 6 Oestrogen vs placebo (in secondary prevention)

Outcome: 6 Venous thromboembolism

\begin{tabular}{|c|c|c|c|c|c|}
\hline Study or subgroup & $\begin{array}{r}\text { Oestrogen } \\
\mathrm{n} / \mathrm{N}\end{array}$ & $\begin{array}{r}\text { Placebo } \\
\mathrm{m} / \mathrm{N}\end{array}$ & $\begin{array}{r}\text { Odds Ratio } \\
\text { M-H,Fixed,95\% CI }\end{array}$ & Weight & $\begin{array}{r}\text { Odds Ratio } \\
\text { M-H,Fixed,95\% C }\end{array}$ \\
\hline SPRT 2002 & $2 / 513$ & 1/504 & $\longrightarrow$ & $20.0 \%$ & $1.97[0.18 .21 .78]$ \\
\hline WEST & 3/337 & $4 / 327$ & & $80.0 \%$ & $0.73[0.16,3.27]$ \\
\hline Total $(95 \% \mathrm{CI})$ & 850 & 831 & - & $100.0 \%$ & $0.97[0.28,3.38]$ \\
\hline \multirow{3}{*}{\multicolumn{6}{|c|}{$\begin{array}{l}\text { Total events: } 5 \text { (Cestrogen), } 5 \text { (Placebo) } \\
\text { Heterogeneity: Chi }=0.48, \text { df }=1 \quad(P=0.49): P^{2}=0.0 \% \\
\text { Test for overal effect: } Z=0.04(P=0.97)\end{array}$}} \\
\hline & & & & & \\
\hline & & & & & \\
\hline & & & $\begin{array}{ccc}0.01 \quad 0.1 & 1 & 10 \\
\text { Favours treatment } & \text { Farours }\end{array}$ & & \\
\hline
\end{tabular}


Analysis 6.7

Comparison 6 Oestrogen vs placebo (in secondary prevention), Outcome 7 Pulmonary embolism

Review: Hormone replacement therapy for preventing cardiovascular disease in postmenopausal women

Comparison: 6 Oestrogen vs placebo (in secondary prevention)

Outcome: 7 Pulmonary embolism

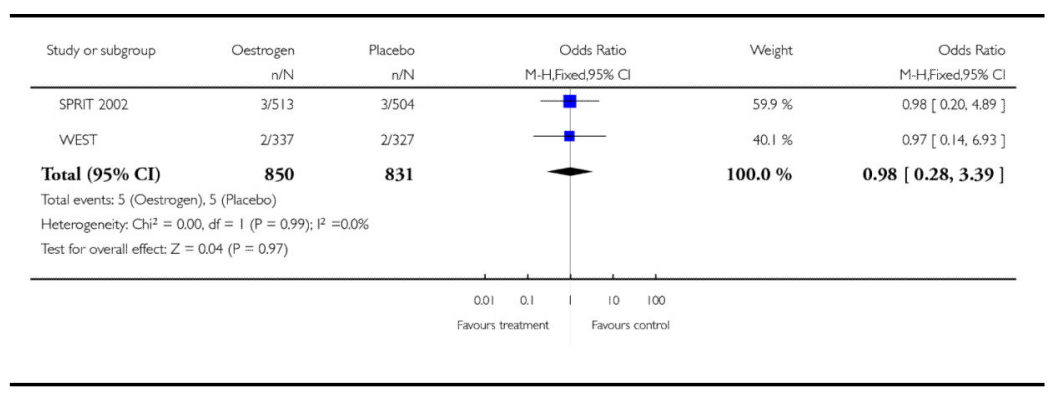

Analysis 7.1

Comparison 7 Combined therapy vs Placebo (in primary or secondary prevention), Outcome 1 Death (all causes)

Review: Hormone replacement therapy for preventing cardiovascular disease in postmenopausal women

Comparison: 7 Combined therapy vs Placebo (in primary or secondary prevention) Outcome: 1 Death (all causes)

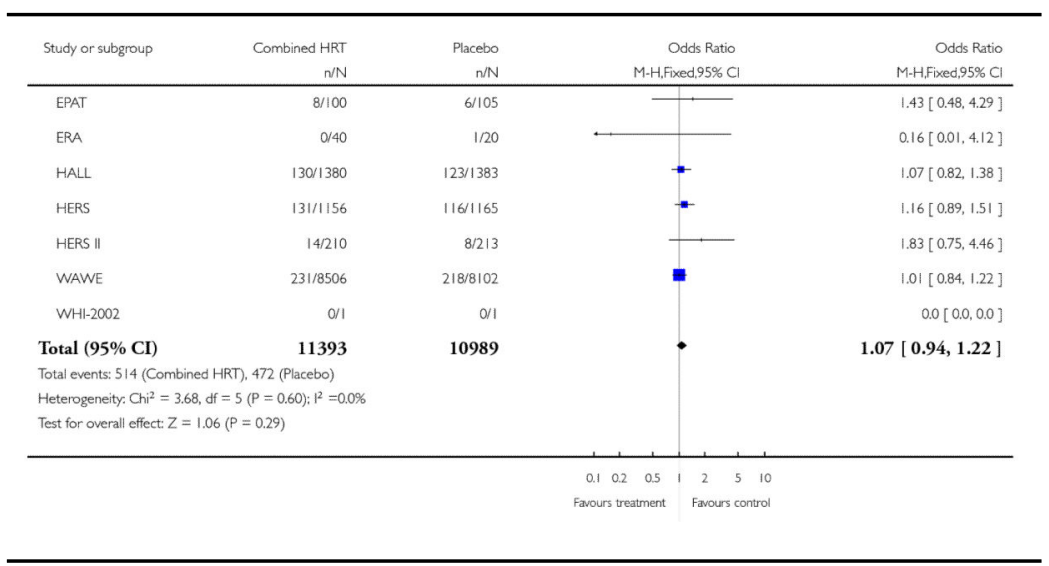




\section{Analysis 7.2}

Comparison 7 Combined therapy vs Placebo (in primary or secondary prevention), Outcome 2 Death

( $\mathrm{CV}$ cause)

Review: Hormone replacement therapy for preventing cardiovascular disease in postmenopausal women

Comparison: 7 Combined therapy vs Placebo (in primary or secondary prevention) Outcome: 2 Death (CV cause)

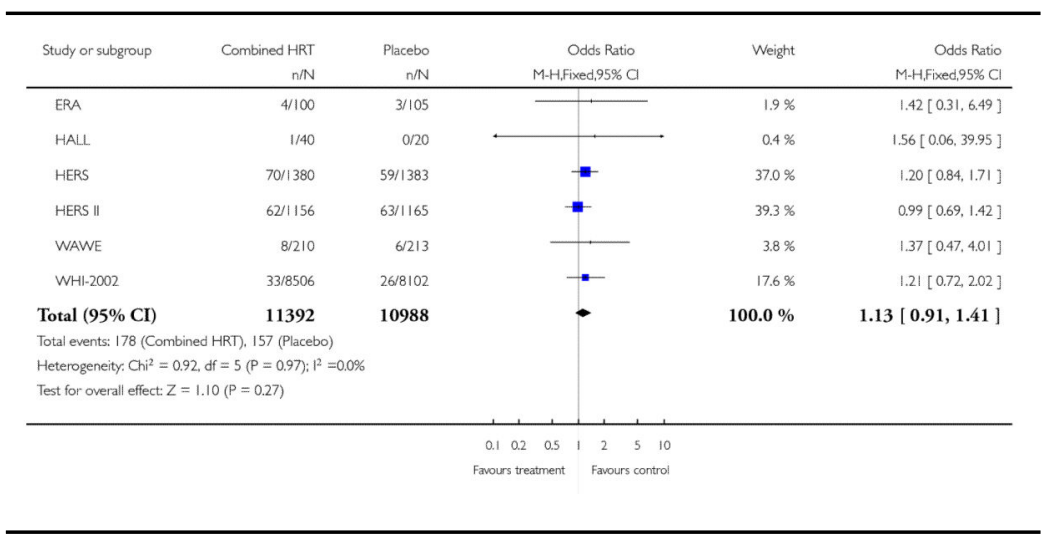

Analysis 7.3

Comparison 7 Combined therapy vs Placebo (in primary or secondary prevention), Outcome 3 Non-fatal MI

Review: Hormone replacement therapy for preventing cardiovascular disease in postmenopausal women

Comparison: 7 Combined therapy vs Placebo (in primary or secondary prevention) Outcome: 3 Non-fatal MI

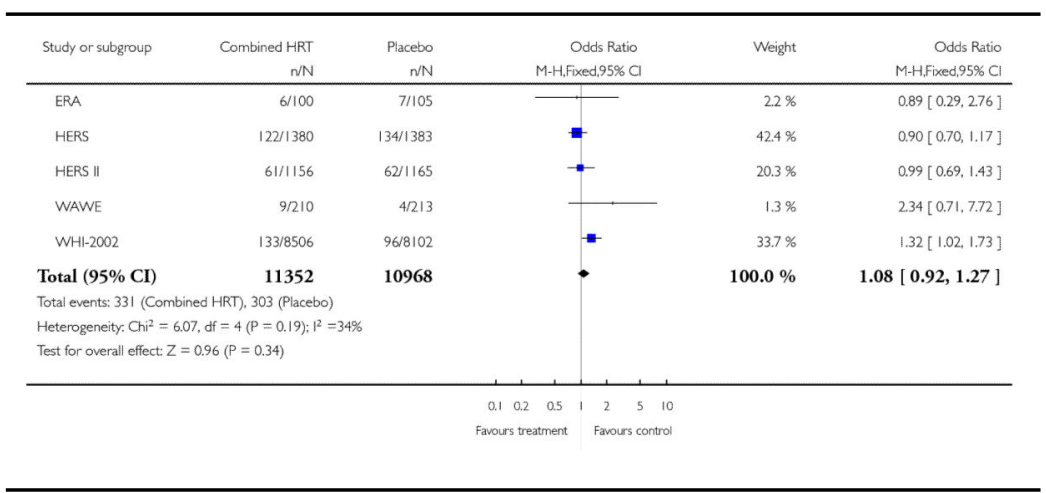


Analysis 7.4

Comparison 7 Combined therapy vs Placebo (in primary or secondary prevention), Outcome 4 Stroke

Review: Hormone replacement therapy for preventing cardiovascular disease in postmenopausal women

Comparison: 7 Combined therapy vs Placebo (in primary or secondary prevention) Outcome: 4 Stroke

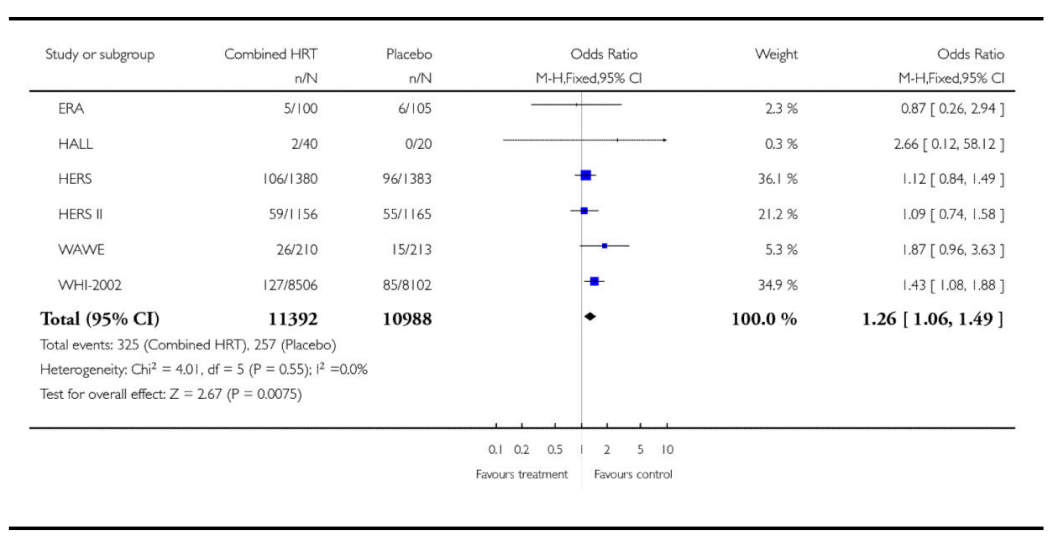

Analysis 7.5

Comparison 7 Combined therapy vs Placebo (in primary or secondary prevention), Outcome 5 Combined CV events and outcomes

Review: Hormone replacement therapy for preventing cardiovascular disease in postmenopausal women

Comparison: 7 Combined therapy vs Placebo (in primary or secondary prevention) Outcome: 5 Combined CV events and outcomes

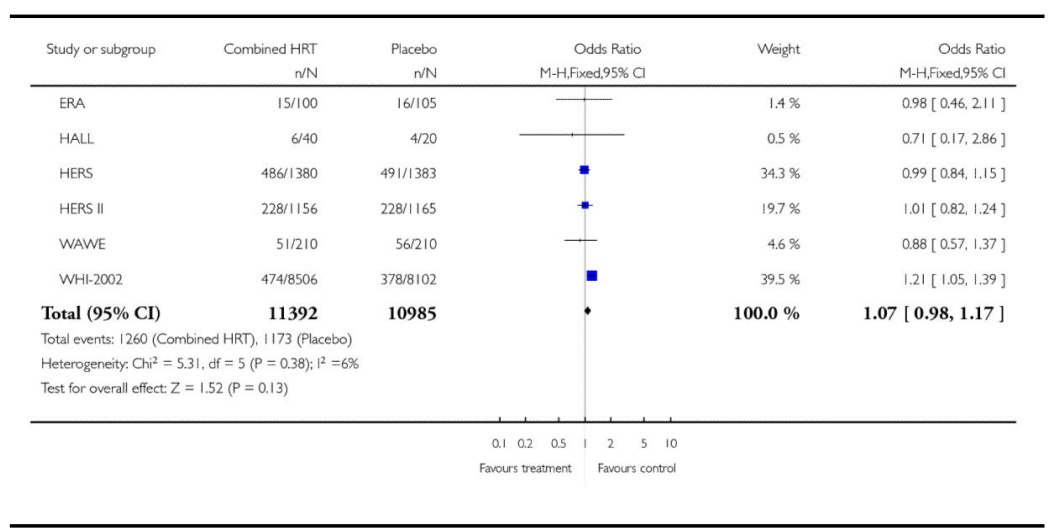


Analysis 7.6

Comparison 7 Combined therapy vs Placebo (in primary or secondary prevention), Outcome 6 Venous thromboembolism

Review: Hormone replacement therapy for preventing cardiovascular disease in postmenopausal women

Comparison: 7 Combined therapy vs Placebo (in primary or secondary prevention)

Outcome: 6 Venous thromboembolism

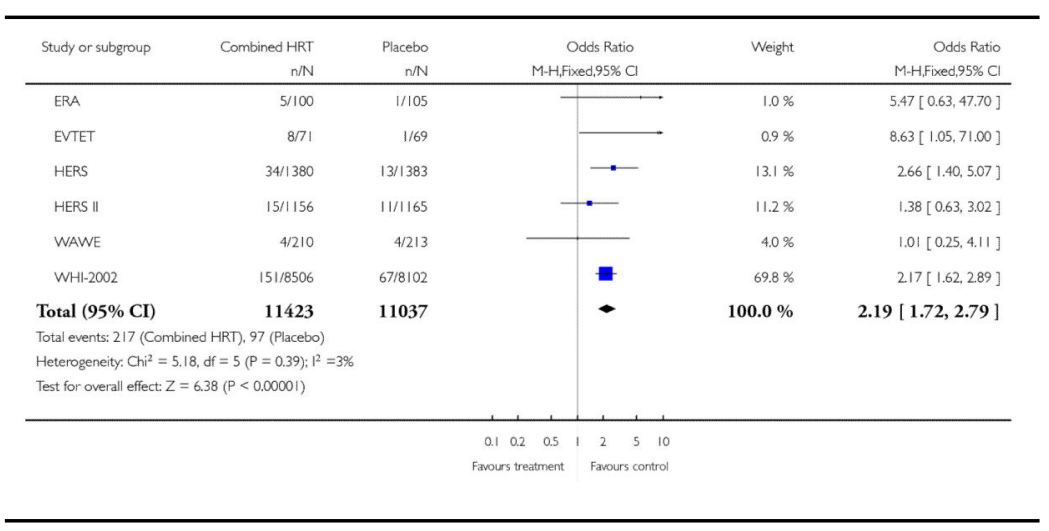

Analysis 7.7

Comparison 7 Combined therapy vs Placebo (in primary or secondary prevention), Outcome 7 Pulmonary embolus

Review: Hormone replacement therapy for preventing cardiovascular disease in postmenopausal women

Comparison: 7 Combined therapy vs Placebo (in primary or secondary prevention) Outcome: 7 Pulmonary embolus

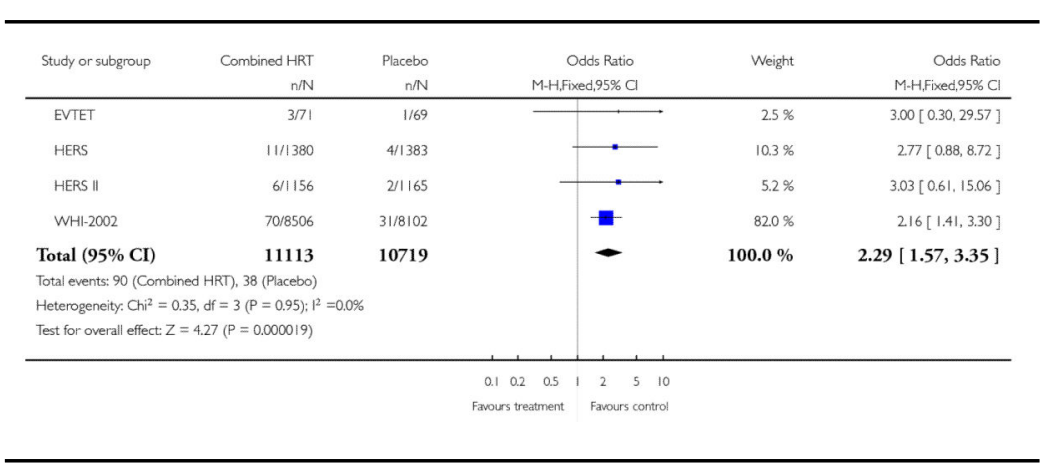




\section{Analysis 8.1}

Comparison 8 Combined therapy vs Placebo (in primary prevention), Outcome 1 Death (all causes)

Review: Hormone replacement therapy for preventing cardiovascular disease in postmenopausal women

Comparison: 8 Combined therapy vs Placebo (in primary prevention)

Outcome: 1 Death (all causes)

\begin{tabular}{|c|c|c|c|c|}
\hline Study or subgroup & $\begin{array}{r}\text { Combined HRT } \\
n / N\end{array}$ & $\begin{array}{r}\text { Placebo } \\
n / N\end{array}$ & $\begin{array}{c}\text { Odds Ratio } \\
\text { M-H,Fixed,95\% CI }\end{array}$ & $\begin{array}{r}\text { Odds Ratio } \\
\text { M-H.Fixed,95\% CI }\end{array}$ \\
\hline \multirow[t]{2}{*}{ WH1-2002 } & $231 / 8506$ & $2 \mid 8 / 8102$ & - & $1.01[0.84,1.22]$ \\
\hline & & & \begin{tabular}{ccc|ccc}
0.1 & 0.2 & 0.5 & 2 & 5 & 10 \\
Farours treatment & Farours control
\end{tabular} & \\
\hline
\end{tabular}

Analysis 8.2

Comparison 8 Combined therapy vs Placebo (in primary prevention), Outcome 2 Death ( $\mathrm{CV}$ cause)

Review: Hormone replacement therapy for preventing cardiovascular disease in postmenopausal women

Comparison: 8 Combined therapy vs Placebo (in primary prevention)

Outcome: 2 Death (CV cause)

\begin{tabular}{|c|c|c|c|c|}
\hline Study or subgroup & $\begin{array}{r}\text { Combined } H R T \\
n / N\end{array}$ & $\begin{array}{r}\text { Placebo } \\
n / N\end{array}$ & $\begin{array}{r}\text { Odds Ratio } \\
\text { M-H, Fixed,95\% Cl }\end{array}$ & $\begin{array}{r}\text { Odds Ratio } \\
\text { M-H,Fixed,95\% CI }\end{array}$ \\
\hline \multirow[t]{2}{*}{ WH1-2002 } & $33 / 8506$ & $26 / 8102$ & - & $1.21[0.72,2.02]$ \\
\hline & & & 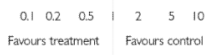 & \\
\hline
\end{tabular}

Analysis 8.3

Comparison 8 Combined therapy vs Placebo (in primary prevention), Outcome 3 Non-fatal myocardial infarction

Review: Hormone replacement therapy for preventing cardiovascular disease in postmenopausal women

Comparison: 8 Combined therapy vs Placebo (in primary prevention)

Outcome: 3 Non-fatal myocardial infarction

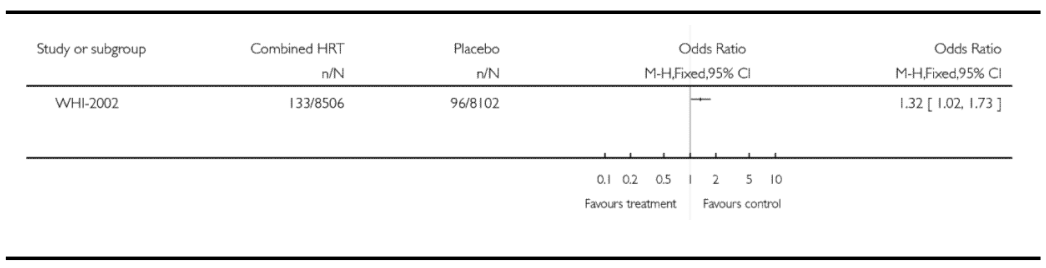




\section{Analysis 8.4}

\section{Comparison 8 Combined therapy vs Placebo (in} primary prevention), Outcome 4 Stroke

Review: Hormone replacement therapy for preventing cardiovascular disease in postmenopausal women

Comparison: 8 Combined therapy vs Placebo (in primary prevention)

Outcome: 4 Stroke

\begin{tabular}{|c|c|c|c|c|}
\hline Study or subgroup & $\begin{array}{r}\text { Combined HRT } \\
n / N\end{array}$ & $\begin{array}{l}\text { Placebo } \\
n / N\end{array}$ & $\begin{array}{c}\text { Odds Ratio } \\
\text { M-H,Fixed,95\% C }\end{array}$ & $\begin{array}{r}\text { Odds Ratio } \\
\text { M-H,Fixed,95\% C }\end{array}$ \\
\hline \multirow[t]{2}{*}{ WH1-2002 } & $127 / 8506$ & $85 / 8102$ & - & $1.43[1.08,1.88]$ \\
\hline & & & 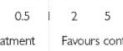 & \\
\hline
\end{tabular}

Analysis 8.5

Comparison 8 Combined therapy vs Placebo (in primary prevention), Outcome 5 Combined CV events and outcomes

Review: Hormone replacement therapy for preventing cardiovascular disease in postmenopausal women

Comparison: 8 Combined therapy vs Placebo (in primary prevention)

Outcome: 5 Combined CV events and outcomes

\begin{tabular}{|c|c|c|c|c|}
\hline Study or subgroup & $\begin{array}{r}\text { Combined HRT } \\
\mathrm{n} / \mathrm{N}\end{array}$ & $\begin{array}{r}\text { Placebo } \\
\mathrm{n} N \mathrm{~N}\end{array}$ & $\begin{array}{r}\text { Odds Ratio } \\
\text { M-H,Fixed,95\% Cl }\end{array}$ & $\begin{array}{r}\text { Odds Ratio } \\
\text { M-H,Fixed,95\% C }\end{array}$ \\
\hline \multirow[t]{2}{*}{ WH1-2002 } & $474 / 8506$ & $378 / 8102$ & + & $1.21[1.05,1.39]$ \\
\hline & & & 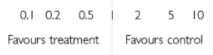 & \\
\hline
\end{tabular}

\section{Analysis 8.6}

Comparison 8 Combined therapy vs Placebo (in primary prevention), Outcome 6 Venous thromboembolism

Review: Hormone replacement therapy for preventing cardiovascular disease in postmenopausal women Comparison: 8 Combined therapy vs Placebo (in primary prevention) Outcome: 6 Venous thromboembolism

\begin{tabular}{|c|c|c|c|c|c|}
\hline \multirow{2}{*}{$\begin{array}{l}\text { Study or subgroup } \\
\text { WH1-2002 }\end{array}$} & \multirow{2}{*}{$\begin{array}{r}\text { Combined HRT } \\
n / N\end{array}$} & \multirow{2}{*}{$\begin{array}{r}\begin{array}{r}\text { Placebo } \\
n / N\end{array} \\
67 / 8102\end{array}$} & \multicolumn{2}{|c|}{$\begin{array}{c}\text { Odds Ratio } \\
\text { M-H.Fixed,95\% CI }\end{array}$} & \multirow{2}{*}{$\begin{array}{r}\text { Odds Ratio } \\
\text { M-H,Fixed,95\% C } \\
2.17[1.62,2.89]\end{array}$} \\
\hline & & & & - & \\
\hline & & & $\begin{array}{c}0.1 \\
0.20 .5 \\
\text { Favours treatment }\end{array}$ & $\begin{array}{ccc}2 & 5 & 10 \\
\text { Favours cortiol }\end{array}$ & \\
\hline
\end{tabular}




\section{Analysis 8.7}

\section{Comparison 8 Combined therapy vs Placebo (in} primary prevention), Outcome 7 Pulmonary embolus

Review: Hormone replacement therapy for preventing cardiovascular disease in postmenopausal women

Comparison: 8 Combined therapy vs Placebo (in primary prevention)

Outcome: 7 Pulmonary embolus

\begin{tabular}{|c|c|c|c|c|c|}
\hline \multirow{2}{*}{$\begin{array}{l}\text { Study or subgroup } \\
\text { WH1-2002 }\end{array}$} & \multirow{2}{*}{$\begin{array}{r}\text { Combined HRT } \\
\mathrm{n} / \mathrm{N}\end{array}$} & \multirow{2}{*}{$\begin{array}{r}\begin{array}{r}\text { Placebo } \\
\text { n/N }\end{array} \\
31 / 8102\end{array}$} & \multicolumn{2}{|c|}{$\begin{array}{c}\text { Odds Ratio } \\
\text { M-H.Fixed,95\% Cl }\end{array}$} & \multirow{2}{*}{$\begin{array}{r}\text { Odds Ratio } \\
\text { M-H,Fixed,95\% C } \\
2.16[1.41,3.30]\end{array}$} \\
\hline & & & & - & \\
\hline & & & $\begin{array}{cc}0.1 \quad 0.20 .5 \\
\text { Favours treatment }\end{array}$ & $\begin{array}{ccc}2 & 5 & 10 \\
\text { Favours control }\end{array}$ & \\
\hline
\end{tabular}

Analysis 9.1

Comparison 9 Combined therapy vs Placebo (in secondary prevention), Outcome 1 Death (all causes)

Review: Hormone replacement therapy for preventing cardiovascular disease in postmenopausal women

Comparison: 9 Combined therapy vs Placebo (in secondary prevention) Outcome: 1 Death (all causes)

\begin{tabular}{|c|c|c|c|c|c|c|}
\hline Study or subgroup & $\begin{array}{r}\text { Combined HRT } \\
\mathrm{n} / \mathrm{N}\end{array}$ & $\begin{array}{r}\text { Placebo } \\
\mathrm{n} / \mathrm{N}\end{array}$ & \multicolumn{2}{|c|}{$\begin{array}{l}\text { Odds Ratio } \\
\text { M-H,Fixed,95\% Cl }\end{array}$} & Weight & $\begin{array}{r}\text { Odds Ratic } \\
\text { M-H.Fixed,95\% C }\end{array}$ \\
\hline ERA & $8 / 100$ & $6 / 105$ & & 9 & $24 \%$ & $1.43[0.48,4.29]$ \\
\hline HALL & $0 / 40$ & 1/20 & & - & $0.9 \%$ & $0.16[0.01,4.12]$ \\
\hline HERS & $130 / 1380$ & $\mid 23 / / 383$ & & $=$ & $48.7 \%$ & $1.07[0.82,1.38]$ \\
\hline HERS \| & $131 / 1156$ & $116 / 165$ & & $=$ & $44.8 \%$ & $1.16[0.89,1.51]$ \\
\hline WAWE & $14 / 210$ & $8 / 213$ & & & $3.2 \%$ & $1.83[0.75,4.46]$ \\
\hline Total $(95 \% \mathrm{CI})$ & 2886 & 2886 & & - & $100.0 \%$ & $1.13[0.95,1.35]$ \\
\hline \multirow{3}{*}{\multicolumn{7}{|c|}{$\begin{array}{l}\text { Total events: } 283 \text { (Combined HRT), } 254 \text { (Placebo) } \\
\text { Heterogeneity: Chi }{ }^{2}=292, \text { df }=4(P=0.57) ; 1^{2}=0.0 \% \\
\text { Test for overall effect } Z=1.36(P=0.17)\end{array}$}} \\
\hline & & & & & & \\
\hline & & & & & & \\
\hline & & & $\begin{array}{c}0.10 .20 .5 \\
\text { Favours treatment }\end{array}$ & $\begin{array}{r}12510 \\
\text { Farours control }\end{array}$ & & \\
\hline
\end{tabular}




\section{Analysis 9.2}

Comparison 9 Combined therapy vs Placebo (in secondary prevention), Outcome 2 Death (CV cause)

Review: Hormone replacement therapy for preventing cardiovascular disease in postmenopausal women

Comparison: 9 Combined therapy vs Placebo (in secondary prevention)

Outcome: 2 Death (CV cause)

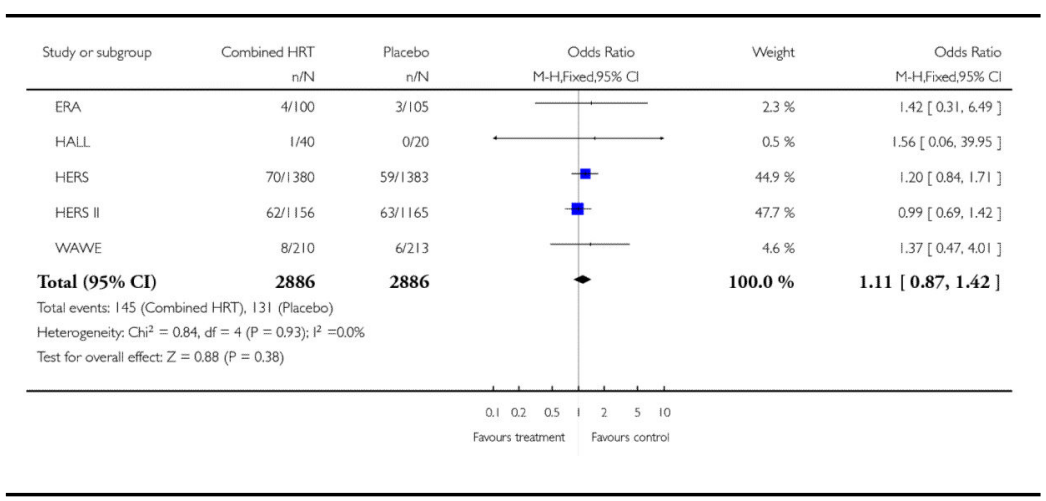

Analysis 9.3

Comparison 9 Combined therapy vs Placebo (in secondary prevention), Outcome 3 Non-fatal myocardial infarction

Review: Hormone replacement therapy for preventing cardiovascular disease in postmenopausal women

Comparison: 9 Combined therapy vs Placebo (in secondary prevention)

Outcome: 3 Non-fatal myocardial infarction

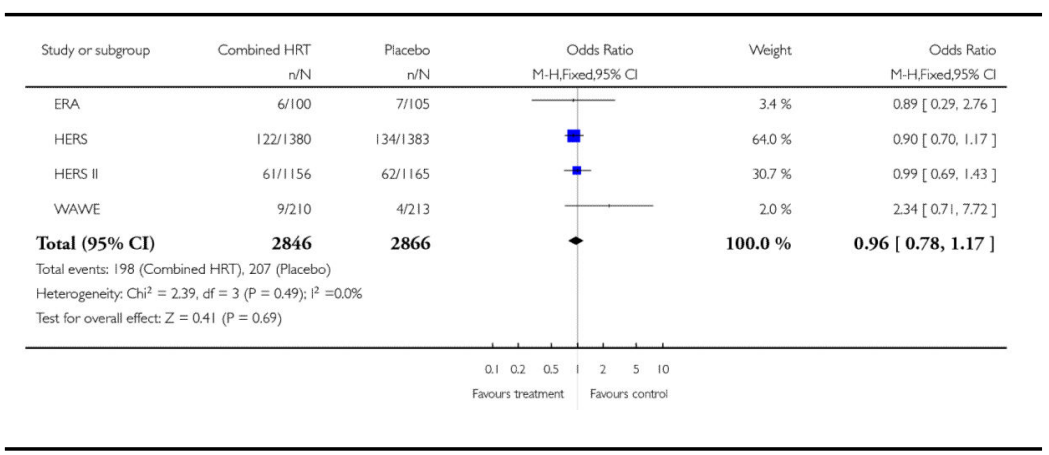




\section{Analysis 9.4}

\section{Comparison 9 Combined therapy vs Placebo (in} secondary prevention), Outcome 4 Stroke

Review: Hormone replacement therapy for preventing cardiovascular disease in postmenopausal women

Comparison: 9 Combined therapy vs Placebo (in secondary prevention)

Outcome: 4 Stroke

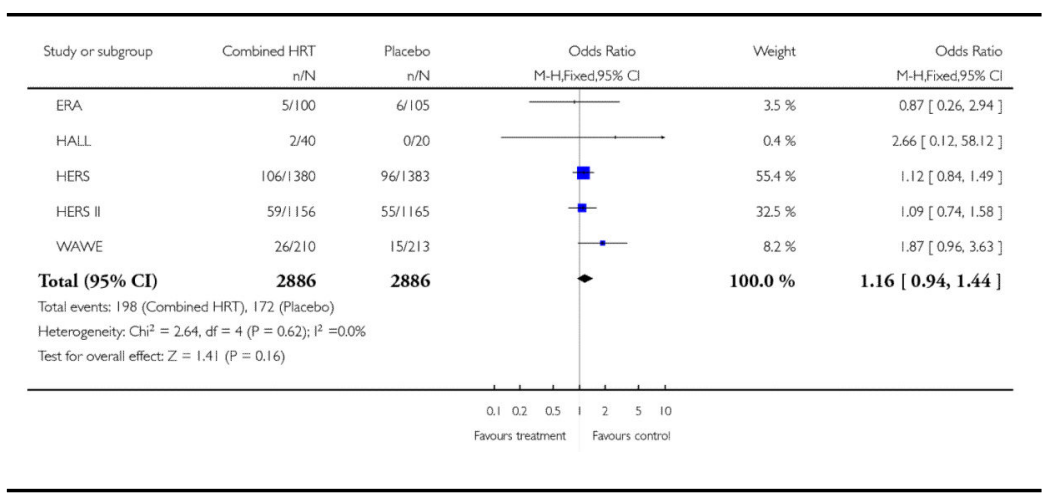

Analysis 9.5

Comparison 9 Combined therapy vs Placebo (in secondary prevention), Outcome 5 Combined CV events

Review: Hormone replacement therapy for preventing cardiovascular disease in postmenopausal women

Comparison: 9 Combined therapy vs Placebo (in secondary prevention) Outcome: 5 Combined CV events

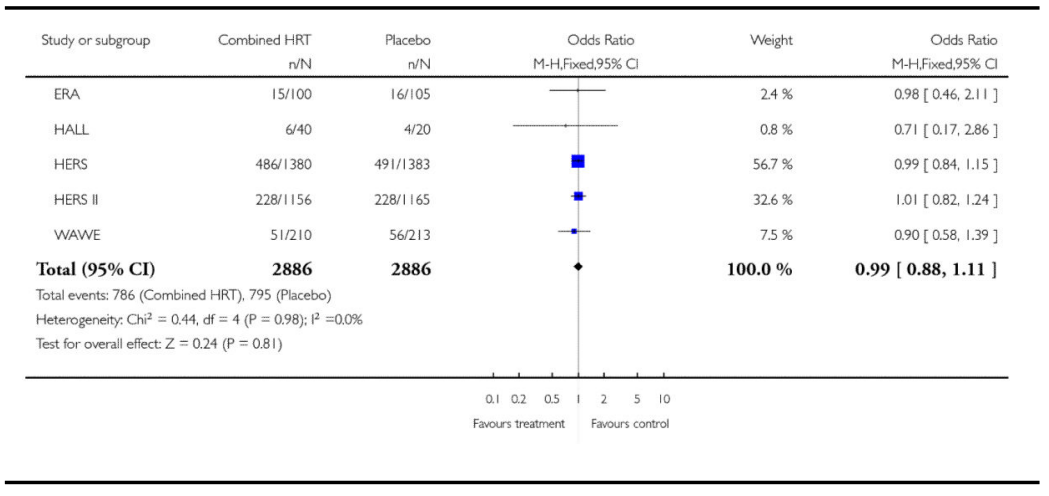




\section{Analysis 9.6}

Comparison 9 Combined therapy vs Placebo (in secondary prevention), Outcome 6 Venous

\section{thromboembolism}

Review: Hormone replacement therapy for preventing cardiovascular disease in postmenopausal women

Comparison: 9 Combined therapy vs Placebo (in secondary prevention)

Outcome: 6 Venous thromboembolism

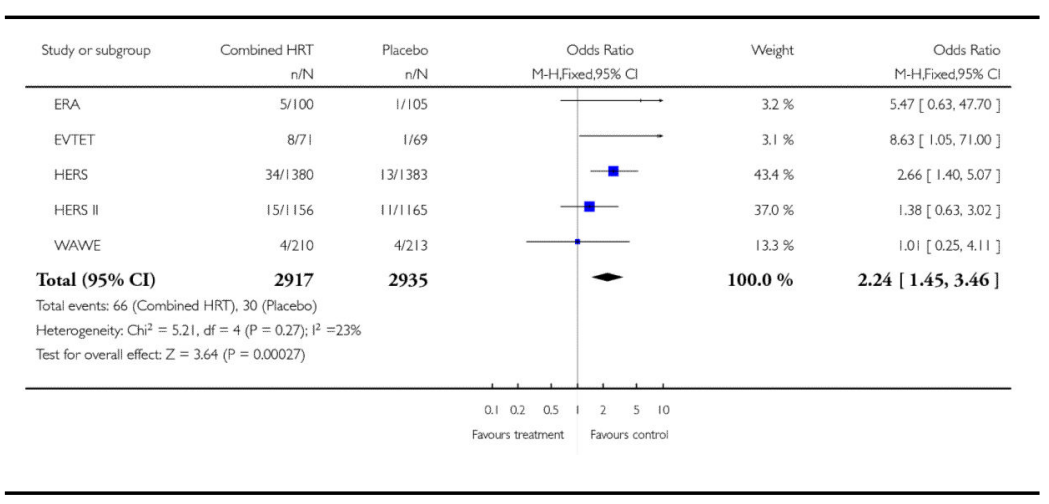

Analysis 9.7

Comparison 9 Combined therapy vs Placebo (in secondary prevention), Outcome 7 Pulmonary embolus

Review: Hormone replacement therapy for preventing cardiovascular disease in postmenopausal women Comparison: 9 Combined therapy vs Placebo (in secondary prevention) Outcome: 7 Pulmonary embolus

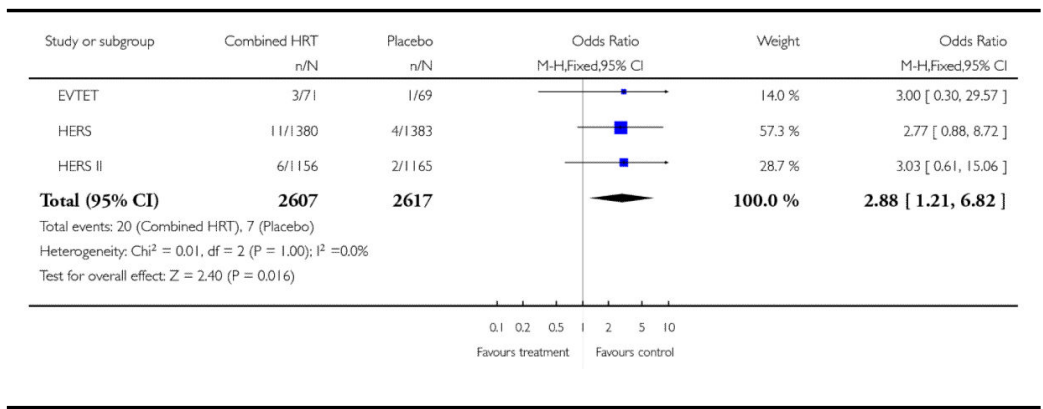

\section{WHAT'S NEW}

Last assessed as up-to-date: 31 January 2005. 


\begin{tabular}{lll}
\hline Date & Event & Description \\
\hline 8 September 2008 & Amended & Converted to new review format. \\
\hline
\end{tabular}

\section{HISTORY}

Protocol first published: Issue 3, 2000

Review first published: Issue 2, 2005

\begin{tabular}{lll}
\hline Date & Event & Description \\
\hline 1 February 2005 & New citation required and conclusions have changed & Substantive amendment \\
\hline
\end{tabular}

\section{References to studies included in this review}

EPAT \{published data only\} . *Hodis HN, Mack WJ, Lobo RA, Shoupe D, Sevanian A, Mahrer PR, et al. Estrogen in the Prevention of Atherosclerosis. A Randomized, Double-Blind, PlaceboControlled Trial. Annals of Internal Medicine. 2001; 135(11):939-53. [PubMed: 11730394]

ERA \{published data only\} . *Herrington DM, Reboussin DM, Bridget Brosnihan K, Sharp PC, Shumaker SA, Snyder TE, et al. Effects of estrogen replacement on the progression of coronaryartery atherosclerosis. New England Journal of Medicine. 2000; 343(8):522-9. [PubMed: 10954759]

Herrington DM, Reboussin DM, Klein KP, Sharp PC, Shumaker SA, Snyder TE, et al. The estrogen replacement and atherosclerosis (ERA) study: study design and baseline characteristics of the cohort. Controlled Clinical Trials. 2000; 21(3):257-85. [PubMed: 10822123]

EVTET \{published data only\} . Hoibraaten E, Arnesen H, Larsen S, Wickstrom E, Sandset PM. Increased risk of recurrent venous thromboembolism during hormone replacement therapy: results of the randomized, double blind, placebo-controlled estrogen in venous thromboembolism trial (EVTET). Thrombosis and Haemostasis. 2000; 84:961-7. [PubMed: 11154141]

HALL \{published data only\} . *Hall G, Pripp U, Schenk-Gustafsson K, Landgren BM. Longterm effects of hormone replacement therapy on symptoms of angina pectoris, quality of life and compliance in women with coronary artery disease. Maturitas. 1998; 28:235-42. [PubMed: 9571599]

HERS \{published data only\} . Grady D, Applegate W, Bush T, Furberg C, Riggs B, Hulley S. Heart and Estrogen/progestin Replacement Study (HERS): design, methods, and baseline characteristics. Controlled Clinical Trials. 1998; 19(4):314-35. [PubMed: 9683309]

*Hulley S, Grady D, Bush T, Furberg C, Herrington D, Riggs B, et al. Randomized trial of estrogen plus progestin for secondary prevention of coronary heart disease in postmenopausal women. JAMA. 1998; 280(7):605-13. [PubMed: 9718051]

Simon JA, Hsia J, Cauley JA, Richards C, Harris F, Fong J, et al. Postmenopausal hormone therapy and risk of stroke: The Heart and Estrogen-progestin Replacement Study (HERS). Circulation. 2001; 103(5):638-42. [PubMed: 11156873]

HERS II \{published data only\} . Grady D, Herrington D, Bittner V, Davidson M, Hlatky M, Hsia J, et al. for the HERS Research Group. Cardiovascular Disease Outcomes During 6.8 Years of Hormone Therapy. Heart and Estrogen/Progestin Replacement Study Follow-up (Hers II). JAMA. 2002; 288(1):49-57. [PubMed: 12090862]

*Hulley S, Furberg C, Barrett Connor E, Cauley J, Grady D, Haskell H, et al. for the HERS Research Group. Noncardiovascular Disease Outcomes During 6,8 Years of Hormone Therapy. Heart and 
Estrogen/Progastin Replacement Study Follow-up (HERS II). JAMA. 2002; 288(1):58-65. [PubMed: 12090863]

SPRIT 2002 \{published data only\} . The ESPRIT Team. Oestrogen therapy for prevention of reinfarction in postmenopausal women: a randomised placebo controlled trial. Lancet. 2002; 360:2001-8. [PubMed: 12504395]

WAWE \{published data only\} . *Waters D, Alderman E, Hsia J, Howard B, Cobb F, Rogers W, et al. Effects of hormone replacement therapy and antioxidant vitamin supplements on coronary atherosclerosis in postmenopausal women. JAMA. 2002; 288:2432-40. [PubMed: 12435256]

WEST \{published data only\} .*Viscoli CM, Brass LM, Kernan WN, Sarrel PM, Suissa S, Horwitz RI. A clinical trial of estrogen-replacement therapy after ischemic stroke. New England Journal of Medicine. 2001; 345(17):1243-9. [PubMed: 11680444]

WHI-2002 \{published data only\} . Roussow JE, Finnegan LP, Harlan WR. The evolution of the Women's Health Inititative: perspective from the NIH. Journal of the American Medical Womens Association. 1995; 50:50-5.

The Womeń s Health Initiative Study Group. Design of the Womeń s Health Initiative Clinical Trial and Observational Study. Controlled Clinical Trials. 1998; 19:61-109. [PubMed: 9492970]

*Writing Group for the Womeń s Health Iniciative Investigators. Risk and Benefits of Estrogen Plus Progestin in Healthy Postmenopausal Women. Principal Results From the Womeń s Health Initiative Randomized Controlled Trial. JAMA. 2002; 288(3):321-33. [PubMed: 12117397]

\section{References to studies excluded from this review}

AITKEN \{published data only\} . Aitken JM, Hart DM, Lindsay R. Oestrogen replacement therapy for prevention of osteoporosis after oophorectomy. BMJ. 1973; 3:515-8. [PubMed: 4741605]

ALOIA \{published data only\}. Aloia JF, Ashok V, Russo L, Sheenan M, Flaster E. The influence of menopause and hormone replacement therapy on body cell mass and body fat mass. American Journal of Obstetrics and Gynecology. 1995; 172:896-900. [PubMed: 7892882]

*Aloia JF, Ashok V, Yeh JK, Ross PL, Flaster E, Dilmanian A. Calcium supplementation with and without hormone replacement therapy to prevent postmenopausal bone loss. Annals of Internal Medicine. 1994; 120:97-103. [PubMed: 8256988]

ANGERER \{published data only\} . Angerer P, Stork S, Kothny W, Schmitt P, von Schacky C. Effect of oral postmenopausal hormone replacement on progression of atherosclerosis: a randomized, controlled trial. Arteriosclerosis, Thrombosis, and Vascular Biology. 2001; 21(2): 262-8.

Barret Connor \{published data only\} . Barrett-Connor E, Grady D, Sashegyi A, Anderson PW, Cox DA, Hoszowski K, et al. for the MORE Investigators. Raloxifene and Cardiovascular Events in Osteoporotic Postmenopausal Women: Four-Year Results From the MORE (Multiple Outcomes of Raloxifene Evaluation) Randomized Trial. JAMA. 2002; 287:847-57. [PubMed: 11851576]

CHART \{published data only\} .*Speroff L, Rowan J, Symons MS, Genant H, Wilborn W. The comparative effect on bone density, endometrium, and lipids of continuous hormones as replacement therapy (CHART Study). JAMA. 1996; 276(17):1397-403. [PubMed: 8892714]

CHRISTENSEN \{published data only\} . Christensen MS, Christiansen C, Transbøl IB. Doseresponse evaluation of cyclic estrogen/gestagen in postmenopausal women: Placebo-controlled trial of its gynecologic and metabolic actions. American Journal of Obstetrics and Gynecology. 1982; 144:873-9. [PubMed: 6756151]

CHRISTIANSEN (a) \{published data only\} . Christiansen C, Riis BJ. 17b-Estradiol and continuous norethisterone: A unique treatment for established osteoporosis in elderly women. The Journal of Clinical Endocrinology and Metabolism. 1990; 71:836-41. [PubMed: 2205624]

CHRISTIANSEN (b) \{published data only . Christiansen C, Christensen P, Rødbro P, Hagen C, Transbøl I. Effect of 1,25-dihydroxy-vitamin D3 $\mathrm{n}$ itself or combined with hormone treatment in preventing postmenopausal osteoporosis. European Journal of Clinical Investigation. 1981; 11:305-9. [PubMed: 6795047] 
CHRISTIANSEN (c) \{published data only . Christiansen C, Christensen MS, McNair P, Hagen C, Stockland K-E, Transbøl IB. Prevention of early postmenopausal bone loss: controlled 2year sudy in 315 normal females. European Journal of Clinical Investigation. 1980; 10:273-9. [PubMed: 6253303]

COOPE \{published data only\}. Coope J. Is oestrogen therapy effective in the treatment of menopausal depression? The Journal of the Royal College of General Practitioners. 1981; 31:134-40. [PubMed: 6268783]

DERMAN \{published data only\} .*Derman RJ, Dawood MY, Stone S. Quality of life during sequential hormone replacement therapy - A placebo-controlled study. International Journal of Fertility. 1995; 40:73-8. [PubMed: 7599662]

GALLAGHER \{published data only\} . Gallagher JC, Kable WT, Goldgar D. Effect of progestin therapy on cortical and trabecular bone: Comparison with Estrogen. American Journal of Medicine. 1991; 90:171-8. [PubMed: 1847582]

GENANT \{published data only\} . Genant HK, Baylnk DJ, Gallagher JC, Harris ST, Steiger P, Herber M. Effect of estrone sulfate on postmenopausal bone loss. Obstetrics and Gynecology. 1990; 76:579-84. [PubMed: 2170884]

HASSAGER \{published data only\} . Hassager C, Christiansen C. Estrogen/gestagen therapy changes soft tissue body composition in postmenopausal women. Metabolism: Clinical \& Experimental. 1989; 38(7):662-5. [PubMed: 2661966]

Hassager C, Riis BJ, Strom V, Guyene TT, Christiansen C. The long-term effect of oral and percutaneous estradiol on plasma renin substrate and blood pressure. Circulation. 1987; 76(4): 753-8. [PubMed: 3308164]

Heckbert 1997 \{published data only\} . Heckbert SR, Weiss NS, Koepsel TD, et al. Duration of estrogen replacementtherapy in relation to the risk of incident myocardial infarction in post menopausal women. Archives of Internal Medicine. 1997; 157:1330-6. [PubMed: 9201007]

JENSEN \{published data only\} . *Jensen J, Riis BJ, Strøm V, Christiansen C. Long-term and withdrawal effects of two different oestrogen-progestogen combinations on lipid and lipoprotein profiles in postmenopausal women. Maturitas. 1989; 11:117-28. [PubMed: 2547137]

LINDSAY \{published data only\} . Lindsay R, Hart DM, Clark DM. The minimum effective dose of estrogen for prevention of postmenopausal bone loss. Obstetrics and Gynecology. 1984; 63(6): 759-63. [PubMed: 6374537]

LUFKIN \{published data only\} .*Lufkin EG, Wahner HW, O'Fallon WM, Hodgson SF, Kotowicz MA, Lane AW, et al. Treatment of postmenopausal osteoporosis with transdermal estrogen. Annals of Internal Medicine. 1992; 117:1-9. [PubMed: 1534476]

MARSLEW \{published data only\} . Marslew U, Overgaard K, Riis BJ, Christiansen C. Two new combinations of estrogen and progestogen for prevention of postmenopausal bone loss: longterm effects on bone, calcium and lipid metabolism, climacteric symptoms, and bleeding. Obstetrics \& Gynecology. 1992; 79(2):202-10. [PubMed: 1309944]

MOLANDER \{published data only\}. Molander U, Milsom P, Ekelund P, Mellsötrm D, Eriksson O. Effect of oral oestriol on vaginal flora and cytology and urogenital symptoms in the postmenopause. Maturitas. 1990; 12:113-20. [PubMed: 2255263]

MUNK-JENSEN \{published data only\} .*Munk-Jensen N, Pors Nielsen S, Obel EB, Bonne Erikson P. Reversal of postmenopausal vertebral bone loss by oestrogen and progestogen: a double blind study. BMJ. 1988; 296:1150-2. [PubMed: 3132244]

Munk-Jensen N, Ulrich LG, Obel EB, Pors Nielsen S, Edwards D, Meinertz H. Continuous combined and sequential estadiol and norethindrone acetate treatment of postmenopausal women: Effect on plasma lipoproteins in a two-year placebo-controlled trial. American Journal of Obstetrics and Gynecology. 1994; 171:132-8. [PubMed: 8030688]

Obel EB, Munk-Jensen N, Svenstrup B, Bnnett P, Micic S, Henrik-Nielsen R, et al. A two-year double-blind controlled study of the clinical effect of combined and sequential postmenopausal replacement therapy and steroid metabolism during treatment. Maturitas. 1993; 16:13-21. [PubMed: 8429800]

NACHTIGALL \{published data only\} . Nachtigall LE, Nachtigall RH, Nachtigall RD, Beckman EM. Estrogen replacement therapyII: A prospective study $n$ the relationship to carcinoma and 
cardiovascular and metabolic problems. Obstetrics and Gynecology. 1979; 54:74-9. [PubMed: 221871]

PEPI \{published data only\} . *The Writing Group for the PEPI Trial. Effects of estrogen/progestin regimens on heart disease risk factors on postmenopausal women. The postmenopausal estrogen/ progestin (PEPI) trial. JAMA. 1995; 273(3):199-208. [PubMed: 7807658]

RESCH \{published data only\} . Resch H, Pietschmann P, Krexner E, Woloszczuk, Willvonseder R. Effects of oneyear hormone replacement therapy on peripheral bone mineral content in patients with osteoporotic spine fractures. Acta Endocinol. 1990; 123:14-18.

RIGGS \{published data only\} . Riggs HL, Seeman E, Hodgson SF, Taves DR, O'Fallon WM. Effect of fluoride/calcium regimen on vertebral fracture occurrence in postmenopausal osteoporosis. New England Journal of Medicine. 1982; 306:446-50. [PubMed: 6276746]

RIIS \{published data only\} . Riis BJ, Thomsen K, Strom V, Christiansen C. The effect of percutaneous estradiol and natural progesterone on postmenopausal bone loss. American Journal of Obstetrics \& Gynecology. 1987; 156(1):61-5. [PubMed: 3541622]

SVENDSEN \{published data only . Svendsen OL, Hassager C, Marslew U, Christiansen C. Changes in calcanean bone mineral occurring spontaneously and during hormone replacement therapy in early postmenopausal women. Scandinavian Journal of Clinical \& Laboratory Investigation. 1992; 52(8):831-6. [PubMed: 1336890]

TONSTAD \{published data only\} . Tonstad S, Ose L, Gørbitz C, Djøseland O, Bard JM, Fruchart JC. Efficacy of sequential hormones replacement therapy in the treatment of hypercholesterolaemia among postmenopausal women. Journal of Internal Medicine. 1995; 238:39-47. [PubMed: 7608645]

WIMALAWANSA \{published data only\}. Wimalawansa SJ. Combined therapy with estrogen and etidronate has an additive effect on bone mineral density in the hip and vertebrae: Four-year randomized study. The American Journal of Medicine. 1995; 99:36-42. [PubMed: 7598140]

\section{References to ongoing studies}

ESPRIT-UK \{unpublished data only\} . Kahn MA, Heagerty AM, Kitchener H, McNamee R, Cherry NM, Hannaford P. Oestrogen and womeń s heart disease. The Quarterly Journal of Medicine. 2000; 93:699-702.

NHLBI \{unpublished data only\} . Ongoing study. 1999. Postmenopausal Hormone Therapy in Unstable Angina.

PHASE \{unpublished data only\} . Ongoing study Starting date of trial not provided. The Papworth HRT Atherosclerosis Survival Enquiry. Contact author for more information

WHISP \{unpublished data only\} . Ongoing study Starting date of trial not provided. Women's hormone intervention secondary prevention study. Contact author for more information

WISDOM \{published data only\} . Vickers RM, Collins N. Progress on the WISDOM trial Womeń s International Study of Long duration ostrogen after the menopause. Climacteric. 2002; 5(Suppl 1):133-4.

Vickers RM, Meade TW, Wilkes HC. Hormone replacement therapy and cardiovascular disease: the case for a randomized controlled trial. Ciba Foundation Symposium. 1995; 191:150-64. [PubMed: 8582195]

\section{Additional references}

Angerer 2002 . Angerer P, Stork S, Kothny W, Schmitt P, von Schacky C. Effect of oral postmenopausal hormone replacement on progression of atherosclerosis: a randomized, controlled trial. Arteriosclerosis, thrombosis, and vascular biology. 2001; 21(2):262-8.

Anon 2004 . Anon HRT. What are women (and their doctors) to do? Lancet. 2004; 364:2069-70. [PubMed: 15589290]

Barret-Connor 1998 . Barret-Connor E, Grady D. Hormone replacement therapy, heart disease, and other considerations. Annual Review of Public Health. 1998; 19(1):55-72. 
Beral 2002 . Beral V, Banks E, Reeves G. Evidence from randomised trials on the long term effects of hormone replacement therapy. Lancet. 2002; 360:942-4. [PubMed: 12354487]

Clarke 2002 . Clarke SC, Kelleher J, Lloyd-Jones H, Slack M, Schofiel PM. A study of hormone replacement therapy in postmenopausal women with ischaemic heart disease: the Papworth HRT atherosclerosis study. BJOG: An International Journal of Obstetrics and Gynaecology. 2002; 109:1056-62. [PubMed: 12269682]

de Klejin 2002 . de Klejin MJJ, van der Schouw YT, Verbeek ALM, Peeters PHM, Banga JD, van der Graaf Y. Endogenous estrogen exposure and cardiovascular mortality risk in postmenopausal women. American Journal of Epidemiology. 2002; 155(4):339-45. [PubMed: 11836198]

Dickersin 1994 . Dickersin K, Scherer R, Lefebvre C. Identifying relevant studies for systematic reviews. BMJ. 1994; 309:1286-91. [PubMed: 7718048]

Fletcher 2002 . Fletcher SW, Colditz GA. Failure of Strogen plus Progestin Therapy for Prevention. JAMA. 2002; 288:366-8. [PubMed: 12117403]

Grady 1992 . Grady D, Rubin SM, Petitti DB, Fox CS, Black D, Ettinger B, et al. Hormone therapy to prevent disease and prolong life in postmenopausal women. Annals of Internal Medicine. 1992; 117(12):1016-37. [PubMed: 1443971]

Grady 2002 . Grady D. A 60 year-old Woman Trying to Discontinue Hormone Replacement Therapy. JAMA. 2002; 287:2130-7. [PubMed: 11966388]

Grodstein 1996 . Grodstein F, Stampfer Mj, Manson JE, Colditz GA, Willet WC, Rosner B, et al. Postmenopausal estrogen and progestin use and the risk of cardiovascular disease. New England Journal of Medicine. 1996; 355:453-61. [PubMed: 8672166]

Hemminki 1997 . Hemminki E, McPherson K. Impact of postmenopausal hormone therapy on cardiovascular events and cancer: pooled data from clinical trials. BMJ. 1997; 315(7101):14953. [PubMed: 9251544]

Hemminki 2000 . Hemminki R, McPherson K. Value of drug-licensing documents in studying the effect of postmenopausal hormone therapy on cardiovascular disease. Lancet. 2000; 355(9203): 566-9. [PubMed: 10683020]

HRT Study Group 2005 . Cochrane HRT Study Group. Long term hormone replacement therapy for perimenopausal and postmenopausal women (Cochrane Protocol). The Cochrane Library. 2003; (2)

Hulley, 1998 . Hulley S, Grady D, Bush T, Furberg C, Herrington D, Riggs B, et al. Heart and Estrogen/Progestin Replacement Study (HERS) Research Group. Randomized trial of estrogen plus progestin for secondary prevention of coronary heart disease in postmenopausal women. JAMA. 1998; 280:605-13. [PubMed: 9718051]

Humphrey 2002 . Humphrey LL, Chan B, Sox HC. Postmenopausal hormone replacement therapy and the primary prevention of Cardiovascular diseases. Annals of Internal Medicine. 2002; 137:273-84. [PubMed: 12186518]

Jadad 1996 . Jadad AR, Moore RA, Carroll D, Jenkinson C, Reynolds DJ, Gavaghan DJ, et al. Assessing the quality of reports of randomized clinical trials: is blinding neccesary? Controlled Clinical Trials. 1996; 17:1-12. [PubMed: 8721797]

Keating 1999 . Keating NL, Cleary PD, Rossi AS, Zaslavsky AM, Ayanian JZ. Use of hormone replacement therapy by postmenopausal women in the United States. Annals of Internal Medicine. 1999; 130(7):545-53. [PubMed: 10189323]

Lawlor 2004 . Lawlor DA, Davey Smith G, Ebrahim S. Socioeconomic Position and Hormone Replacement Therapy Use: Explaining the Discrepancy in Evidence From Observational and Randomized Controlled Trials. American Journal of Public Health. 2004; 94:2149-54. [PubMed: 15569967]

Lefebvre 1996 . Lefebvre, C.; McDonald, S. Development of a sensitive search strategy for reports of randomized controlled trials in EMBASE; Fourth International Cochrane Colloquium; Adelaide. 1996;

Petitti 1994 . Petitti DB. Coronary heart disease and estrogen replacement therapy. Can complicance bias explain the results of observational studies? Annals of Epidemiology. 1994; 4(2):115-8. [PubMed: 8205277] 
Pettiti 2002 . Pettiti DB. Hormone Replacement Therapy for Prevention. More Evidence, More Pessimism. JAMA. 2002; 288:99-101. [PubMed: 12090868]

Schulman 2002 . Schulman SP, Thiemann DR, Ouyang P, Chandra NC, Schulman DS, Reis SE, et al. Effects of acute hormone therapy on recurrent ischemia in postmenopausal women with unstable angina. The Journal of the American College of Cardiology. 2002; 39:231-7.

Stampfer 1991 . Stampfer MJ, Colditz GA. Estrogen replacement therapy and coronary heart disease: a quantitative assessment of the epidemiologic evidence. Preventive Medicine. 1991; 20:47-63. [PubMed: 1826173]

Vastag 2002 . Vastag B. Hormone Replacement Therapy Falls Out of Favor With Expert Committee. JAMA. 2002; 287:1923-4. [PubMed: 11960518]

Wenger 2000 . Wenger NK. Hormonal and Nonhormonal Therapies for the Postmenopausal Woman: What is the Evidence for Cardioprotection? The American Journal of Geriatric Cardiology. 2000; 9(4):204-9. [PubMed: 11416567]

White 2002 . White C. Second long term HRT trial stopped early. BMJ. 2002; 325:987. [PubMed: 12411340]

*Indicates the major publication for the study 


\section{PLAIN LANGUAGE SUMMARY}

Hormone therapy has no heart-protective benefit to healthy postmenopausal women or to those with heart disease

The authors analysed the data from the 10 clinical trials - two involved healthy women and eight involved women with heart disease. Altogether the trials included about 24,000 women who had been randomly assigned to take either hormones or placebos (dummy pill) every day for approximately five years. The authors found no evidence that hormone therapy provides heart-related benefits to postmenopausal women with or without heart disease. Rather, women taking hormones had a higher incidence of non-fatal heart attacks, stroke, and blood clots in the leg and lung than the women taking placebos. 